\title{
A numerical model for ocean ultra-low frequency noise: Wave-generated acoustic-gravity and Rayleigh modes
}

\author{
Fabrice Ardhuin, Thibaut Lavanant, and Mathias Obrebski \\ Ifremer, Laboratoire d'Océanographie Spatiale, Plouzané, France \\ Louis Marié \\ Ifremer, Laboratoire de Physique des Océans, UMR6523 CNRS/Ifremer/IRD/UBO Plouzané, France \\ Jean-Yves Royer and Jean-François d'Eu \\ Domaines Océaniques, CNRS, Plouzané, France \\ Bruce M. Howe and Roger Lukas \\ School of Ocean and Earth Science and Technology, University of Hawaii at Manoa, Honolulu, Hawaii \\ Jerome Aucan \\ Laboratoire d'Etudes en Géophysique et Océanographie Spatiale, Toulouse, France
}

(Received 9 July 2012; revised 8 April 2013; accepted 9 April 2013)

\begin{abstract}
The generation of ultra-low frequency acoustic noise $(0.1$ to $1 \mathrm{~Hz})$ by the nonlinear interaction of ocean surface gravity waves is well established. More controversial are the quantitative theories that attempt to predict the recorded noise levels and their variability. Here a single theoretical framework is used to predict the noise level associated with propagating pseudo-Rayleigh modes and evanescent acoustic-gravity modes. The latter are dominant only within $200 \mathrm{~m}$ from the sea surface, in shallow or deep water. At depths larger than $500 \mathrm{~m}$, the comparison of a numerical noise model with hydrophone records from two open-ocean sites near Hawaii and the Kerguelen islands reveal: (a) Deep ocean acoustic noise at frequencies 0.1 to $1 \mathrm{~Hz}$ is consistent with the Rayleigh wave theory, in which the presence of the ocean bottom amplifies the noise by 10 to $20 \mathrm{~dB}$; (b) in agreement with previous results, the local maxima in the noise spectrum support the theoretical prediction for the vertical structure of acoustic modes; and (c) noise level and variability are well predicted for frequencies up to $0.4 \mathrm{~Hz}$. Above $0.6 \mathrm{~Hz}$, the model results are less accurate, probably due to the poor estimation of the directional properties of wind-waves with frequencies higher than 0.3 Hz. @ 2013 Acoustical Society of America. [http://dx.doi.org/10.1121/1.4818840]
\end{abstract}

PACS number(s): 43.30.Nb, 43.30.Ma, 43.30.Pc [JAC]

Pages: $3242-3259$

\section{INTRODUCTION}

Ultra-low frequency acoustic noise (ULF, 0.1 to $1 \mathrm{~Hz}$ ) has been observed from shallow water ${ }^{1}$ to all depths of the deep ocean. ${ }^{2,3}$ At large depths, noise has been associated with seismic pseudo-Rayleigh waves that propagate over thousands of kilometers, ${ }^{3,4}$ from oceans to land. The term "pseudo-Rayleigh" is also used for the same modes generated by earthquakes, and it emphasizes the effect of the water layer in which the motion is a superposition of obliquely propagating sound waves. ${ }^{5}$ In the crust, the amplitude of motion decays with depths, similar to usual Rayleigh waves, with a combination of shear and compression waves that gives zero tangential stress on the ocean bottom. For simplicity we will omit the "pseudo" and, even in the oceans, we will use the term "Rayleigh" waves. These waves propagate slower than the shear wave speed in the crust and usually faster than the speed of sound in water. ${ }^{6}$ These are clearly seismo-acoustic waves that propagate from oceans to land, where they produce typical vertical ground surface displacements (VGSDs) on the order of a few micrometers, which generally dominate seismic records. That propagation is not yet fully understood and the horizontal gradients in crust properties and water depth may be responsible for strong reflection and scattering.

Close to the ocean surface, ULF noise is consistent with forced gravity waves, ${ }^{2}$ which are also influenced by the compressibility of water, and that we will call acoustic-gravity (A-G) modes. Both types, Rayleigh (R) and A-G motions are forced by near-surface nonlinear hydrodynamic interactions of ocean surface gravity waves (OSGWs). ${ }^{5,7,8}$

The objective of the present paper is to verify this theory quantitatively using pressure records from oceanic locations. This effort is a logical extension of the validation of seismic noise models at land locations. ${ }^{9,10}$ Although land-based seismometers have been very useful to verify the modeled patterns of noise sources in space and time,${ }^{11}$ the noise models still suffer from inaccurate seismic attenuation and propagation, so that model-data mismatches are difficult to interpret. The interpretation of acoustic noise in the ocean is more direct, because it avoids the propagation from ocean to continent, which is difficult to model.

Also, previous measurements have shown that the acoustic spectrum could reveal details about the different Rayleigh modes that compose the seismo-acoustic noise field at large depths, and noise measurements can be used to infer bottom 
properties including sediment shear speeds. ${ }^{12}$ These modes are difficult to observe in land records, probably due to mode conversion as the Rayleigh waves propagate in shallower water.

The present work is also an extension of previous attempts $^{13-17}$ at modeling ULF noise in the ocean. The novelty here is the use of a state-of-the-art numerical wave model that should be able to represent some of the wave directional parameters. In particular Duennebier et al. ${ }^{17}$ estimated wave directional properties from measurements at the ocean bottom, based on a theory which considers sound generated by waves in an ocean of infinite depth. ${ }^{13,14}$ That analysis is not consistent with the noise generation theory successfully applied to seismic noise, ${ }^{9,10}$ and other authors have shown the importance of the ocean bottom for underwater sound with frequencies from 0.1 to $0.7 \mathrm{~Hz}{ }^{18-20}$

Here we take into account the effect of the ocean bottom, and a spatially and temporally varying random OSGW field. This OSGW field is provided by a numerical spectral wave model. ${ }^{21,22}$ In that respect, we extend the work of Webb ${ }^{19}$ who also used a Rayleigh wave theory ${ }^{8}$ but who, like Duennebier et al., ${ }^{17}$ used a OSGW directional spectrum parameterized from the local wind speed only. The particularly novel aspect of the present paper is the use of both near surface measurements, dominated by A-G modes that are useful to verify the local source magnitude, and the use of deep water pressure records, which are more common but more difficult to interpret quantitatively.

The structure of the paper is as follows. The noise generation theory is briefly reviewed in Sec. II, and the numerical model is described in Sec. III. The accuracy of noise sources is established in Sec. IV for noise frequencies up to $0.4 \mathrm{~Hz}$. Application of the same model in Secs. V and VI to measurements at 550 and $4718 \mathrm{~m}$ depth reveals the importance of sediment layers that significantly changes the Rayleigh mode structures, and suggests that numerical models for OSGWs are not faithfully representing the directional wave spectrum variability at OSGW frequencies above $0.4 \mathrm{~Hz}$. Conclusions and perspectives follow in Sec. VII.

\section{NOISE GENERATION THEORY}

Longuet-Higgins ${ }^{7}$ and Hasselmann ${ }^{8}$ considered the acoustic and seismic response of a water layer of constant depth $h$, mean density $\rho_{w}$, and sound speed $c_{w}$, coupled to a homogeneous solid half space, the crust, of density $\rho_{c}$ with compression and shear wave speeds $c_{c p}$ and $c_{c s}$. This Pekeris wave guide is excited by OSGWs. While Hasselmann assumed that $h$ is much larger than the wavelength of the OSGWs, corrections appropriate to any depth have been given by Ardhuin and Herbers. ${ }^{5}$ The theory is extended in Appendix A, with the addition of a homogeneous sediment layer of thickness $h_{s}$ and compression and shear wave velocities $c_{s p}$ and $c_{s s}$.

Gravity and acoustic wave motion in the water column is nearly irrotational, and the associated velocity potential can be expanded in powers of the surface slope

$$
\phi=\phi_{1}+\phi_{2}+\ldots
$$

The first order potential $\phi_{1}$ is the sum of linear waves for which compressibility effects are negligible. ${ }^{7}$
At the second order, the nonlinear interaction of any pair of linear surface gravity wave trains of frequencies $f$ and $f^{\prime}$ and wavenumber vectors $\mathbf{k}$ and $\mathbf{k}^{\prime}$ has an effect that is equivalent to a surface pressure $\hat{p}_{2 \text {,surf }}$ with frequency $f_{s}$ $=f+f^{\prime}$ and wavenumber $\mathbf{K}=\mathrm{k}+\mathrm{k}^{\prime}$. For nearly opposite vectors $\mathbf{k}$ and $\mathbf{k}^{\prime}$ with nearly the same magnitude, then $f_{s}=f+f^{\prime} \simeq 2 f$ and $|\mathbf{K}| \ll|\mathbf{K}|$. This surface pressure field thus has a phase speed $2 \pi\left(f+f^{\prime}\right) /|\mathbf{K}|$ that can match the horizontal phase speed of acoustic waves in the ocean.

The linearized acoustic wave equation is ${ }^{7}$

$$
c_{w}^{2} \nabla^{2} \phi_{2}=\frac{\partial^{2} \phi_{2}}{\partial t^{2}}
$$

with $\nabla$ the three-dimensional gradient operator. Seeking solutions that propagate horizontally with a wavenumber vector $\mathbf{K}$, and thus a phase speed $c=\left(2 \pi f_{s}\right) / K$, gives solutions that have a vertical wavenumber $l$

$$
l=K \sqrt{\frac{c^{2}}{c_{w}^{2}}-1}
$$

These solutions are sound waves, that propagate at an angle $\arctan (K / l)$ relative to the vertical, determined by $f_{s}$ and $K$. Vertical propagation corresponds to $K=0$, for which $c$ is infinite, and horizontal propagation corresponds to $c=c_{w}$.

For $c<c_{w}, l$ becomes imaginary and the corresponding acoustic modes are evanescent. In the limit of very large $K$ (corresponding to $c \ll c_{w}$ ), we find $l=\mathrm{i} K$ which corresponds to gravity waves in deep water. As a result, the acoustic field is a superposition of both propagating and evanescent modes, all driven by the surface gravity waves.

The velocity potential for the noise $\phi_{2}$ must also satisfy the combined kinematic and dynamic boundary condition which is identical to the surface boundary condition for gravity waves [Eq. (4.2) of Hasselmann ${ }^{23}$ ], which may be written as $^{8}$

$$
\left(\frac{\partial^{2}}{\partial t^{2}}+g \frac{\partial}{\partial z}\right) \phi_{2}=-\frac{1}{\rho w} \frac{\partial \hat{p}_{2, \text { surf }}}{\partial t},
$$

where the equivalent surface pressure $\hat{p}_{2 \text {,surf }}$ is a complicated expression of the frequency directional spectrum of OSGWs. The pressure in the water column is then given by

$$
p=p_{1}-\rho_{w} \frac{\partial \phi_{2}}{\partial t}-\frac{1}{2} \rho_{w}\left|\nabla \phi_{1}\right|^{2},
$$

where $p_{1}$ and $\phi_{1}$ are the lowest order (linear) pressure and velocity potential associated with the surface gravity waves.

The frequency-directional ocean wave spectrum $F(f, \theta)$ can be expressed in terms of a directional distribution $M(f, \theta)$ and a frequency (heave) spectrum $E(f)$, such that $F(f, \theta)=E(f) M(f, \theta)$. From $M(f, \theta)$, we define the integral $I(f)$ that represents the net effect of all waves traveling in opposite directions,

$$
I(f)=\int_{0}^{2 \pi} M(f, \theta) M(f, \theta+\pi) \mathrm{d} \theta
$$


which corresponds to the usual ${ }^{24}$ definition, and is a factor of 2 larger than the definition used in other studies. ${ }^{5,10,25}$ As described in Sec. III, $F(f, \theta)$ and thus $I(f)$ can be computed by numerical wave models forced by winds, but these models have not yet been validated in terms of $I(f)$, which is one of our goals.

When the OSGWs are in deep water, (i.e., $k h>\pi$ ) the expression for $\hat{p}_{2 \text {,surf }}$ simplifies. In particular, for $K \simeq 0$, the power spectrum of $\hat{p}_{2, \text { surf }}$ is

$$
F_{p 2, \text { surf }}\left(\mathbf{K} \simeq 0, f_{s}\right)=\rho_{w}^{2} g^{2} f E^{2}(f) I(f),
$$

where $f_{s}=2 f$. Because $\mathbf{K}$ is a horizontal vector, $F_{p 2 \text {,surf }}$ is a three-dimensional spectral density with S.I. units of $\mathrm{Pa}^{2} \mathrm{~m}^{2}$ s.

As discussed by many others, ${ }^{10,17,24}$ the integral $I(f)$ is responsible for a very large variability in acoustic and seismic noise levels. Ardhuin et al. ${ }^{10}$ have classified the sea states that generate noise in three classes. Class I corresponds to waves generated by the local winds, i.e., the wind sea, having a broad enough distribution in directions to produce a strong noise level by themselves. This is typically the dominant class at high frequencies. ${ }^{17,24,25}$ Class II corresponds to wave reflection off the coast, with reflected energy that can have directions opposite to the incoming wave direction. Class II generally dominates at the lowest frequencies $\left(f_{s}<0.1 \mathrm{~Hz}\right)$, but it is relatively weak due to the small reflection of natural shorelines. The loudest noise sources are in fact found in conditions of opposing swells, or wind sea and swell, of the same frequency, which defines class III. Such events are very few at the lowest frequencies, for $f_{s}<0.12 \mathrm{~Hz}$.

In $4720 \mathrm{~m}$ depth around Hawaii, only for acoustic frequencies above $0.4 \mathrm{~Hz}$ is the noise level well correlated with the local wind. ${ }^{17}$ This probably corresponds to a general transition from class II and III at $f_{s}<0.4 \mathrm{~Hz}$, for which noise has little relation with the local wind, to class I for $f_{s}>0.4 \mathrm{~Hz}$. At all oceanic locations, this transition is expected to occur for an acoustic frequency less than or around $0.5 \mathrm{~Hz}$.

\section{A. Noise in an unbounded ocean}

In this case, the noise pressure field associated with the velocity potential $\phi_{2}$ is a solution to Eq. (4),

$$
p_{2}=\int \frac{\hat{p}_{2}\left(\mathbf{K}, f_{s}\right)}{1-i g l / \omega_{s}^{2}} \mathrm{e}^{\mathrm{i}[-l z+\Theta(\mathbf{K}, s)]} \mathrm{d} \mathbf{K} \mathrm{d} f_{s}+\text { c.c. }
$$

where c.c. denotes the complex conjugate of the first term, and the phase function of interacting wave trains is defined by

$$
\Theta(\mathrm{K}, s)=\left[\mathrm{K} \cdot \mathbf{x}-2 \pi f_{s} t\right],
$$

where $\mathbf{x}$ is the horizontal position vector.

The integral in Eq. (8) can be separated into propagating and evanescent modes. 5,16

\section{Propagating or "homogeneous" modes, $c>c_{w}$}

In this range, we shall neglect $\mathrm{g}|l| w_{s}^{2}$, which is less than 0.1 for OSGW periods less than 180 s (i.e., a frequency of
$0.005 \mathrm{~Hz}$ and a wavelength of $40 \mathrm{~km}$ ) because it is bounded by the ratio between the deep water OSGW speed and sound speed.

We obtain the frequency spectrum of the propagating modes by summing on only the low wavenumber regions (labeled $\mathrm{P}+\mathrm{S}, \mathrm{S}$, and $\mathrm{R}$ in Fig. 1),

$$
F_{p 2, p}\left(f_{s}\right)=\int_{K<\omega_{s} / c_{w}} F_{p 2, \text { surf }}\left(\mathbf{K}, f_{s}\right) \mathrm{d} \mathbf{K} .
$$

For this range of wavenumbers $\left|k-k^{\prime}\right|<K<\omega_{s} / c_{w}$, so that $2 f\left|f-f^{\prime}\right|<g /\left(4 \pi^{2}\right)\left|k-k^{\prime}\right|$ gives $\left|f-f^{\prime}\right|<g /\left(2 \pi c_{w}\right)$ which is close to $0.001 \mathrm{~Hz}$. Typical ocean wave spectra have a relative frequency half-width $\sigma_{f} / f$ that is between 0.03 for swells
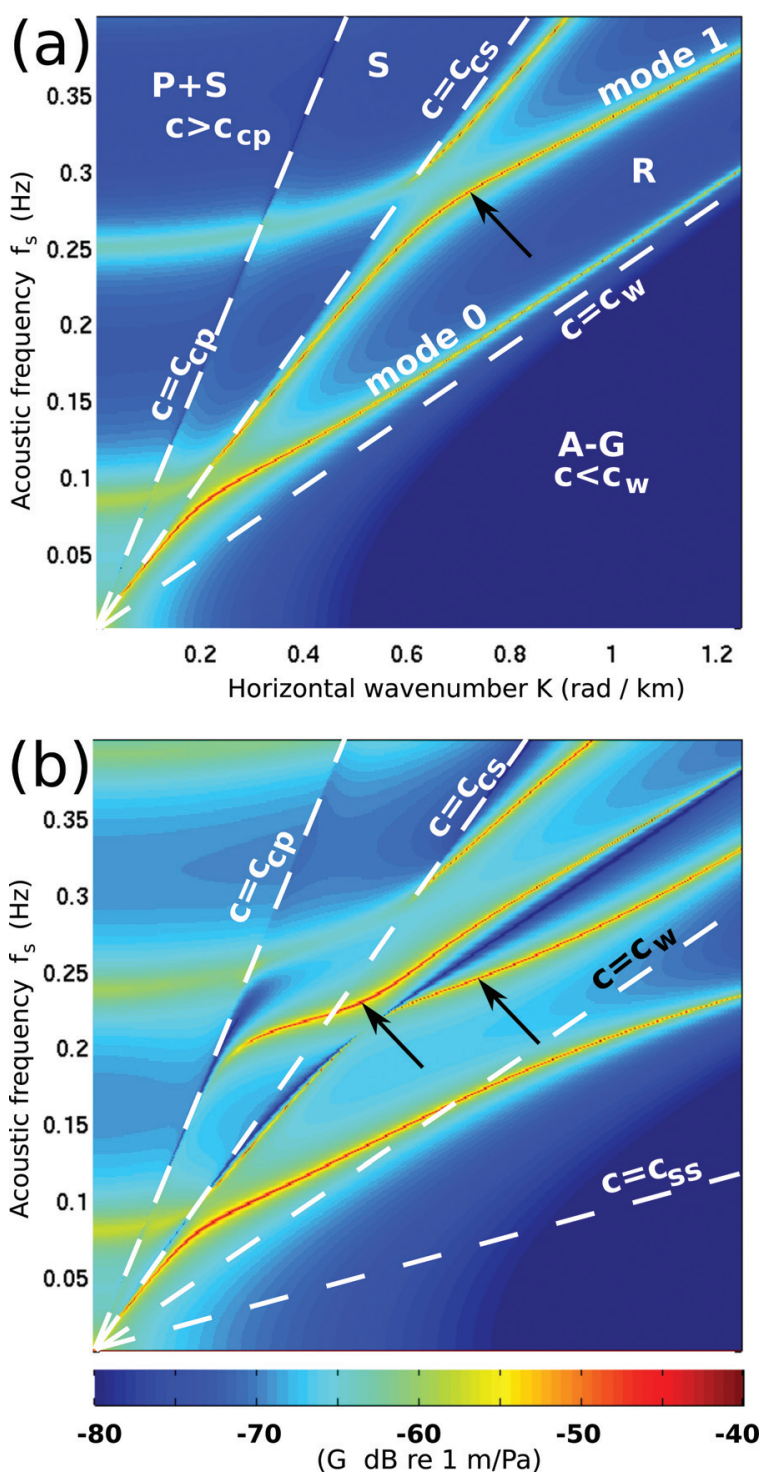

FIG. 1. Surface pressure to ground displacement transfer function $G$. $G$ is estimated for two different sets of properties of the sediment and crust. (a) $h=4440 \mathrm{~m}, c_{w}=1.47 \mathrm{~km} / \mathrm{s}, \rho_{w}=1026 \mathrm{~kg} / \mathrm{m}^{3}, c_{c p}=5.2 \mathrm{~km} / \mathrm{s}, c_{c s}=3.0 \mathrm{~km} / \mathrm{s}$, $\rho_{c}=3300 \mathrm{~kg} / \mathrm{m}^{3}$, (b) with the addition of a $700 \mathrm{~m}$ thick sediment layer, where $c_{s p}=2 \mathrm{~km} / \mathrm{s}, c_{s s}=0.6 \mathrm{~km} / \mathrm{s}, \rho_{s}=1700 \mathrm{~kg} / \mathrm{m}^{3}$. Arrows point to some of the pairs $\left(K, f_{s}\right)$ for which the amplification coefficient $c_{1}$ and $c_{2}$ are maxima. In (a) the arrow corresponds to the peak of mode 1 , mode 0 being the lowest branch where $G$ is singular. The white dotted lines separate the four domains: Body waves $(\mathrm{P}+\mathrm{S})$, mixed body and evanescent waves $(\mathrm{S})$, Rayleigh waves (R), and A-G modes. 
and 0.07 for wind seas,${ }^{26}$ making $E(f) \simeq E\left(f^{\prime}\right)$ a good approximation for typical wave frequencies, larger than $0.05 \mathrm{~Hz}$. The wave spectrum is thus broad enough for us to use the approximation $\mathbf{K}=0$, so that the spectral density $F_{p 2 \text {,surf }}$ is given by Eq. (7), and can be taken out of the integral in Eq. (9). The acoustic spectrum simplifies as

$$
F_{p 2, p}\left(f_{s}\right)=\frac{\pi \omega_{s}^{2}}{c_{w}^{2}} \rho_{w}^{2} g^{2} f E^{2}(f) I(f) .
$$

This is identical to the expression given by Lloyd ${ }^{14}$ and consistent with the more general expression, which accounts for surface tension, used by Farrell and Munk. ${ }^{24}$ We note that this contribution to the noise power spectrum is homogeneous, independent of depth.

\section{Evanescent or "inhomogeneous" modes, $c<c_{w}$}

The pressure associated with the A-G modes is the other part of the integral in Eq. (9), for $K>2 \pi f_{s} / c_{w}$. The imaginary wave number $l$ gives a vertical attenuation of the power spectrum by a factor $\mathrm{e}^{-2 l l / z}$. With that attenuation we may, for large enough depths, consider that only modes with $K \ll k$ contribute to the result. Hence $F_{p 2 \text {,surf }}\left(\mathbf{K}, f_{s}\right)$ $\simeq F_{p 2 \text {,surf }}\left(\mathbf{K}=0, f_{s}\right)$. This is only valid up to a maximum wavenumber $K_{\max }=\varepsilon k$. For numerical applications we will use $\varepsilon=0.2$, the corresponding vertical wavenumber $l$ is approximately equal to $\mathrm{i} K_{\max }$.

With this approximation we have,

$$
\begin{aligned}
F_{p 2, e}\left(f_{s, z}\right) & =F_{p 2, \text { surf }}\left(\mathbf{K}=0, f_{s}\right) 2 \pi \int_{w_{s} / c_{w}}^{K_{\text {mas }}} K \mathrm{e}^{2|l| z} \mathrm{~d} K \\
& =F_{p 2, \text { surf }}\left(\mathbf{K}=0, f_{s}\right) 2 \pi \int_{0}^{K_{\max }}|l| \mathrm{e}^{2|l| z} \mathrm{~d}|l| \\
& =\frac{\pi}{2 z^{2}} \rho_{w}^{2} g^{2} f\left[1-(1-u) \mathrm{e}^{u}\right] E^{2}(f) I(f),
\end{aligned}
$$

where $u=2 z K_{\max }$ and a missing factor $(1-u)$ in Ref. 5 has been added, making this expression exactly the same as the incompressible result given by Cox and Jacobs. ${ }^{2}$

The full solution, without these approximations, is given in Appendix B. Equation (11) explains the observed decay of the noise level near the surface, ${ }^{2}$ which is proportional to $z^{-2}$, and is consistent with other analyses. ${ }^{15,16}$

\section{B. Acoustic waves over an elastic bottom}

Acoustic waves are reflected by the bottom and sea surface, which leads to an amplification of the noise level. Hasselmann ${ }^{8}$ determined the noise field by inverting the matrix $\mathrm{N}$ that expresses the surface and bottom boundary conditions, relating the noise amplitude to the forcing amplitude (see Appendix A for details). Away from wavenumbers at which $\mathrm{N}$ is singular, the power spectrum of the $\operatorname{VGSD} \delta$ is given by

$$
F_{\delta}\left(\mathbf{K}, f_{s}\right)=|G|^{2} F_{p 2 \text {,surf }}\left(\mathbf{K}, f_{s}\right),
$$

where $G$ is a transfer function, proportional to $\operatorname{det}(\mathbf{N})^{-1}$ (Appendix A).
The function $G$ is shown in Fig. 1 for typical sea water and crust properties. Away from singularities, the transfer function $T_{\mathrm{PN}}$ defined by Kibblewhite and $\mathrm{Wu}^{15}$ should be equal to $|G|^{2}$. The $\left(K, f_{s}\right)$-plane is separated into four domains by the three characteristic velocities $c_{w}<c_{c s}<c_{c p}$. From slow to fast, these domains correspond to A-G waves that are evanescent in the water and crust, Rayleigh waves, that are evanescent in the crust only, shear waves (S), and compression $(\mathrm{P})$ waves.

For any water depth $h$ and acoustic frequency $f_{s}$ there exists at least one wavenumber $K_{j}\left(f_{s}\right)$ for which the matrix $\mathbf{N}$ is singular, i.e., $\operatorname{det}\left[\mathbf{N}\left(f_{s}, K_{j}\left(f_{s}\right)\right)\right]=0$. This wavenumber corresponds to a horizontal phase speed $c=2 \pi f_{s} / K_{j}\left(f_{s}\right)$ in the range $c<c_{c s}$ or $c<c_{s s}$ (region $\mathrm{R}$ in Fig. 1), thus corresponding to evanescent shear waves in the crust or sediments. This singularity defines the dispersion relation of the Rayleigh mode number $j$ (see domain R in Fig. 1). Due to the singularity, the energy of the near-singular Rayleigh modes grows linearly with the propagation distance, ${ }^{8}$ for as long as the forcing remains. This gives a variance of the pressure that increases linearly with propagation distance, ${ }^{15}$ and which is not just a function of the local sea state. The magnitude of the ground displacement or bottom pressure depends on the bottom properties, and can be characterized by the coupling coefficients $a_{j}$ and $a_{\mathrm{PB}, j}$ defined in Appendix A and shown in Fig. 2(c).

The pressure field in the water column can also be estimated with the same method (see Appendix A). This is illustrated in Fig. 3 with the transfer function for the pressure power at the sea bottom. It appears clearly that modes with fast horizontal speeds, near $K=0$, can be amplified by up to $20 \mathrm{~dB}$; these are in the body waves domain and are due to the forcing of the acoustic modes near their node at the surface. Although a single reflection from the bottom only expected to increase the noise level by $3.5 \mathrm{~dB}$ for a typical amplitude reflection coefficient of 0.5 , the interference at the surface is responsible for the much larger amplification, which only occurs for particular frequencies. On average the noise level is increased by $10 \mathrm{~dB}$ for the noise components with $K<\omega_{s} / c_{c p}$. Since this region of the wavenumber plane only contains $8 \%$ of the area in the region $K<\omega_{s} / c_{w}$, these body waves contribute little to the overall noise. Indeed, the most important amplifications are found in the Rayleigh wave domain, with singularities along the Rayleigh wave dispersion curves. Because the free Rayleigh modes have zero pressure at the surface and a pressure power profile that is proportional to $\sin ^{2}(l z)$, the surface pressure is very strongly amplified toward the bottom. As a result, the pressure response is dominated by these Rayleigh modes except very close to the surface, and the motion and associated pressure field are identical to those of free Rayleigh or Scholte waves. We may thus compute the amplitudes of pressure $p_{R}$ in the water column from the ground displacement $\delta$ at $z=-h$,

$$
p_{R}(z)=\rho_{w} \delta\left(2 \pi f_{s}\right)^{2} \frac{\sin (l z)}{l \cos (l h)} .
$$

As detailed in Appendix A, the addition of a sediment layer changes the dispersion relations that can now have 

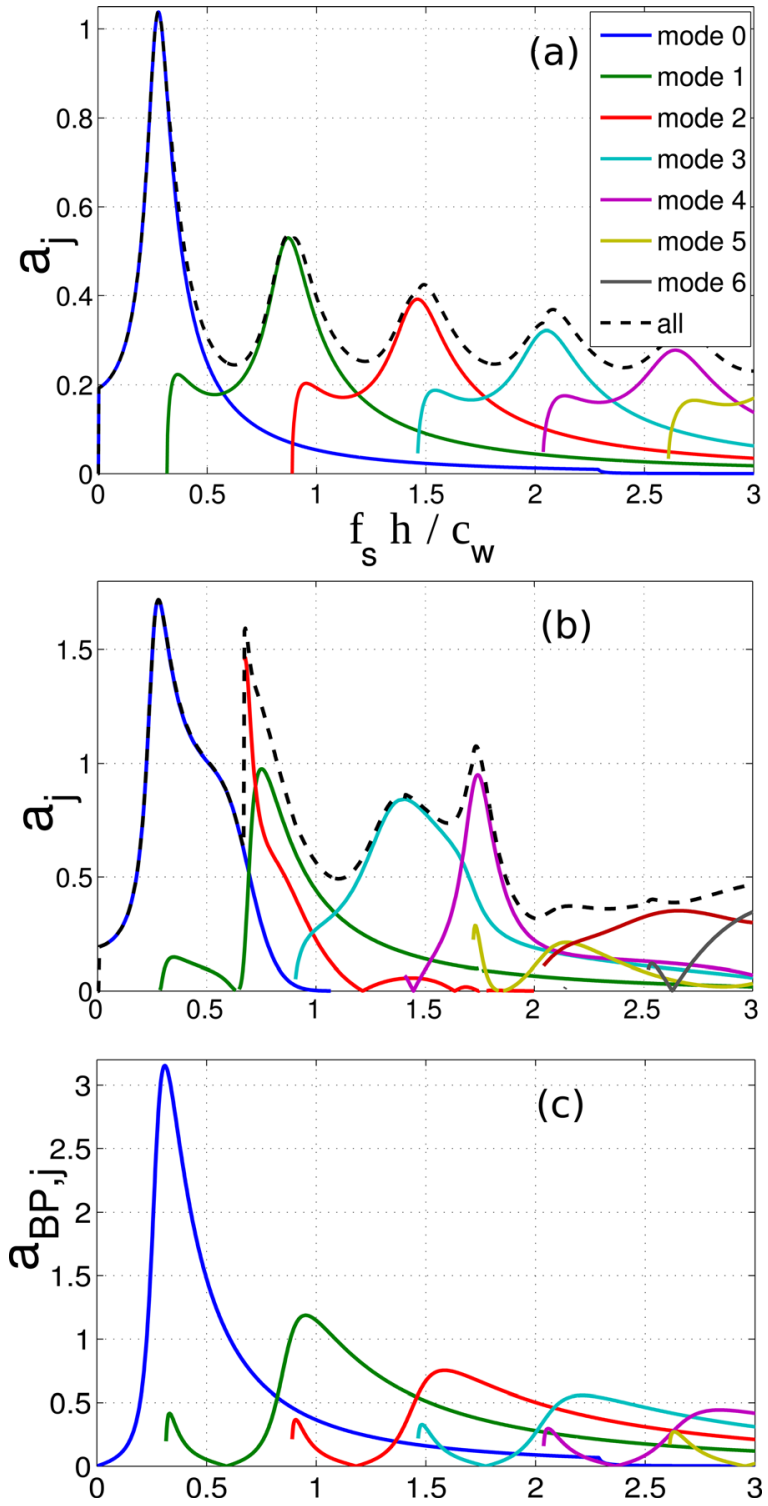

FIG. 2. (Color online) Dimensionless amplification coefficients $a_{j}$ for ground displacement [Eq. (14)]. The amplitude of the peaks depends on the impedance ratio of the sea water and crust, or sediment. In panel (a), we used $\rho_{w}=1026 \mathrm{~kg} / \mathrm{m}^{3}, \quad \rho_{c}=3300 \mathrm{~kg} / \mathrm{m}^{3}, \quad c_{w}=1.47 \mathrm{~km} / \mathrm{s}, \quad c_{c p}=5.2 \mathrm{~km} / \mathrm{s}$, $c_{c s}=3 \mathrm{~km} / \mathrm{s}$. In panel (b) a sediment layer of thickness $h_{s}=700 \mathrm{~m}$ and the water depth is $h=4440 \mathrm{~m}$. The sediment properties are $c_{s p}=2 \mathrm{~km} / \mathrm{s}$, $c_{s s}=0.6 \mathrm{~km} / \mathrm{s}$, and $\rho_{s}=1700 \mathrm{~kg} / \mathrm{m}^{3}$. In (c) the dimensionless coefficient $a_{\mathrm{PB}, j}$ for the bottom pressure is shown for the case without sediments.

phase speeds between the sediment shear wave velocity $c_{s s}$ and the crust compression wave velocity $c_{c p}$. This is illustrated in Fig. 1(b). As a result, the amplification of the surface pressure into ground motion (VGSD) can be strongly modified via the coefficient $a_{j}$ shown in Fig. 2(b). In particular new modes can appear, and the maximum amplification factor of existing modes can shift to different frequencies. In the example chosen here, in the presence of the sediment layer, the amplitude of mode $j=0$ has a hump at $f_{s} h / c_{w} \simeq 0.5$ visible in Fig. 2(b). This hump corresponds to a frequency $f_{s}=0.17$ and wavenumber $K=0.7 \mathrm{rad} / \mathrm{km}$ where the mode speed falls below the sound speed in water, and the Rayleigh waves become Scholte waves, that are also evanescent in the water column. As a result the mode amplitude falls to negligible levels near $f_{s} h / c_{w} \simeq 1$.
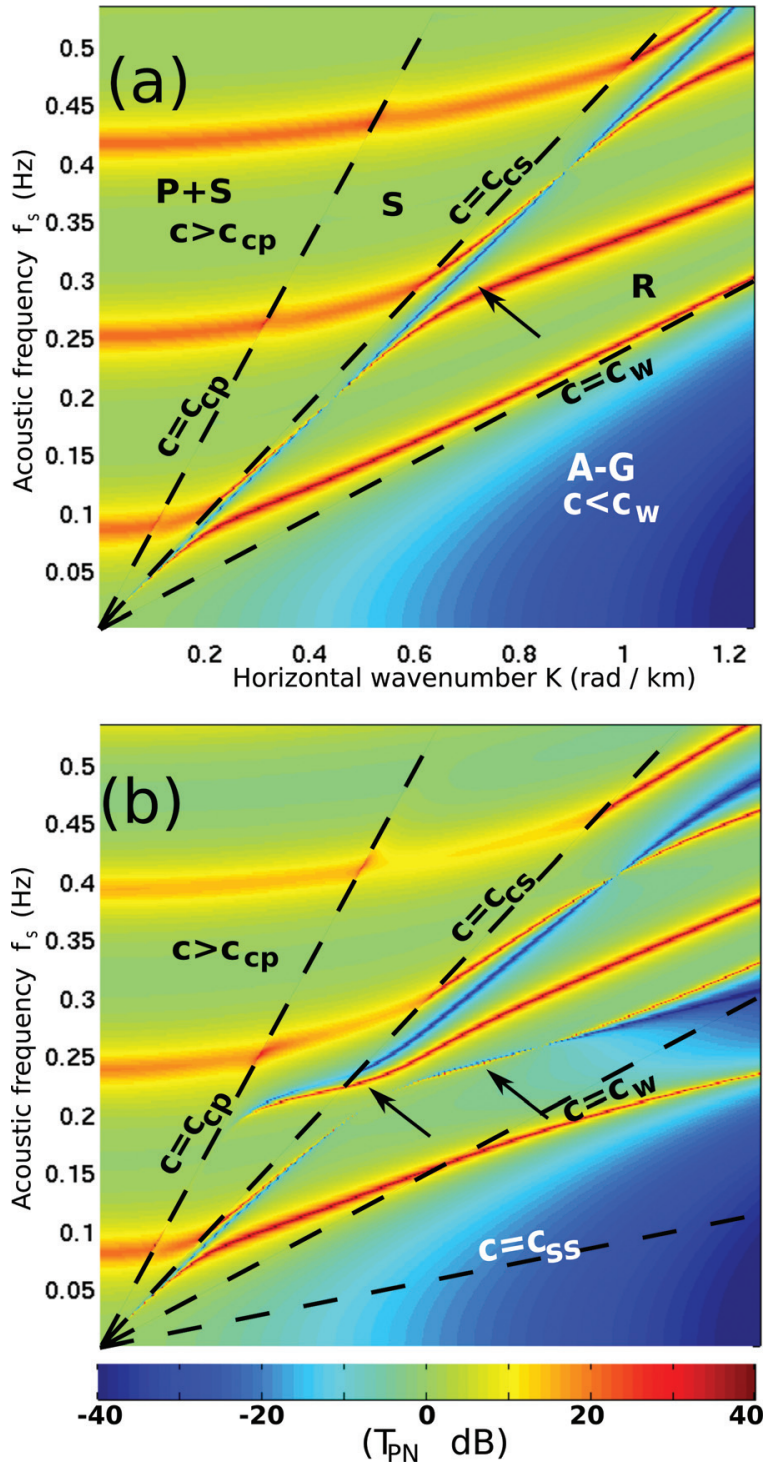

FIG. 3. Same format as Fig. 1, now showing the Transfer function $T_{\mathrm{PN}}$ from the surface pressure spectrum, to the ocean bottom pressure spectrum.

Because the OSGW spectrum varies spatially, the noise solution is better expressed as a sum of local sources. The local rate of increase of the VGSD spectrum is

$$
S_{\mathrm{DF}, j}\left(f_{s}\right)=\frac{4 \pi^{2} f_{s} a_{j}^{2}}{c_{\mathrm{cs}}^{5} \rho_{s}^{2}} F_{p}\left(\mathbf{K} \simeq 0, f_{s}\right),
$$

where $a_{j}$ are the dimensionless coefficients shown in Fig. 2 and defined in Appendix A. The clear maxima of $a_{j}$ correspond to the quarter-wavelength resonance typical of organ pipes. ${ }^{7,8}$ For vertical sound propagation, these maxima occur at values of the acoustic wavelength to water depth ratio $f_{s} h / c_{w}$ that are exactly $1 / 4,3 / 4 \ldots$. This vertical propagation corresponds to the case of seismic body waves. ${ }^{5}$

Here the water pressure and ground motion are dominated by Rayleigh modes that correspond, in the water, to a superposition of upward and downward oblique acoustic waves, hence the peaks of $a_{j}$ correspond to a vertical wavelength equal to $1 / 4,3 / 4 \ldots$ of the water depth. This vertical wavelength is larger than the acoustic wavelength by a factor 
$l \mid \sqrt{\left(K^{2}+l^{2}\right)}$, and the peaks of $a_{j}$, that give the frequencies at which the ground motion is maximum, are shifted by the same factor, pushing them to $f_{s} h / c_{w} \simeq 0.28,0.87, \ldots$ with exact values depending on the sediment or crust properties. Likewise, the amplitudes of the peaks depend on the impedance ratio of the sea water and crust. Hence the peak amplitude increases with $\rho_{s} c_{c s} /\left(\rho_{w} c_{w}\right)$. For example, a $c_{c s}=2800 \mathrm{~m} \mathrm{~s}^{-1}$, gives a maximum of 0.88 for $a_{0}$ instead of 1.03 for $c_{c s}=3000 \mathrm{~m} \mathrm{~s}^{-1}$. In terms of bottom pressure, this shift is even larger, as shown by the shape of the corresponding $a_{\mathrm{PB}, j}$ coefficients in Fig. 2(c).

We may now estimate the VGSD power spectrum for each mode $j$, at an observation point with longitude $\lambda$ and latitude $\phi$, and time $t$. This is the sum of Rayleigh wave sources over the entire ocean, ${ }^{8,10}$

$$
F_{\delta, j}\left(\lambda, \phi, f_{s}, t\right)=\int_{\Omega} \frac{S_{\mathrm{DF}}\left(f_{s}, t-\tau\right)}{R_{E} \sin \Delta} \mathrm{e}^{-\alpha \Delta R_{E}} \mathrm{~d} \Omega,
$$

with $R_{E}$ the Earth radius, $\mathrm{d} \Omega$ the elementary ocean area, and $\tau$ the travel time at the group speed. For practical applications $\tau$ is typically less than $30 \mathrm{~min}$ and it can be ignored because the sea state and thus the noise source $S_{\mathrm{DF}}$ do not change much on this time scale. The denominator $\left(R_{E} \sin \Delta\right)$ is the geometrical spreading factor for wave energy that follows geodesics on the sphere, replacing the distance $\left(R_{E} \Delta\right)$ used in flat Earth models. ${ }^{8}$ The spatial attenuation $\alpha$ is usually written as

$$
\alpha=2 \pi f_{s} /(U Q),
$$

where $U$ and $Q$ are, respectively, the group speed of the Rayleigh wave and the non-dimensional quality factor that represents the attenuation per wave cycle. The large value of $\alpha$, typically $1 \times 10^{-6} \mathrm{~m}^{-1}$ or $0.004 \mathrm{~dB} / \mathrm{km}$ (i.e., an e-folding distance of $1000 \mathrm{~km}$ ) justifies neglecting the absorption of sound in sea water, which is at least 1 order of magnitude smaller.

The corresponding pressure spectrum is finally

$$
F_{p, j}\left(z, f_{s}\right)=\left[\rho_{w}\left(2 \pi f_{s}\right)^{2} \frac{\sin \left(l_{j} z\right)}{l_{j} \cos \left(l_{j} h\right)}\right]^{2} F_{\delta, j}\left(f_{s}\right) .
$$

The net effects of all modes is obtained by summing the power spectra over all modes $j$.

If the attenuation is large compared to the relative spatial variation of the noise source then we may assume the source to be spatially uniform giving,

$$
F_{\delta, j}\left(f_{s}\right)=L_{\mathrm{att}} S_{\mathrm{DF}}\left(f_{s}\right),
$$

with an attenuation scale that is the product of the noise period, group speed, and quality factor,

$$
L_{\text {att }}=T U Q \text {. }
$$

\section{THE NUMERICAL NOISE MODEL}

Our numerical noise model consists of two parts. The first part is a state-of-the-art wave model that is used to estimate the wave directional spectra $F(f, \theta)$ at any location on the ocean surface, using winds that vary in space and time. For the sake of simplicity in the notations, the dependence of $F(f, \theta)$ on the horizontal position and time is not written explicitly. Because the model propagates the wave energy and computes the wave growth and decay that slowly adjust the wave field to the forcing wind, the variability of $F(f, \theta)$ is more realistic than the empirical models ${ }^{17,19}$ that define $F(f, \theta)$ from the local wind only. Our wave model also includes bathymetry, shoreline, varying currents, varying sea ice, and even small icebergs that act as breakwaters in the Southern Ocean. ${ }^{27}$

The second part of the model determines the noise source and performs the noise propagation. It takes the $F(f, \theta)$ wave spectra resulting from the first part, and simply uses Eqs. (14) and (16), which are applied for discrete frequencies $f_{s}$ that correspond to twice the wave model frequencies $f$. This is the simplest possible model, assuming a spherically symmetric Earth with a uniform crust and possibly one sediment layer between the crust and water. This is much simpler than the discretized vertical layering used by Webb. ${ }^{19}$ This simplicity makes for an easier analysis of the results. This model is thus a simple conversion of the seismic noise model of Ardhuin et al. ${ }^{10}$ to compute pressure in the water column. A more robust model would be obtained by expressing directly the noise source in terms of pressure variance at the bottom instead of ground displacement, avoiding the singularities in Eq. (16) that appear at frequencies for which $\cos (l h) \simeq 0$. Our choice was motivated by the desire to compare the ground motion and pressure records.

Our wave model is a global implementation of version 4.07 of the WAVEWATCH III ${ }^{(\mathrm{R})}$ numerical framework. ${ }^{21}$ Specific to our simulations are the use of recent physical parameterizations for wind-wave generation and dissipation, ${ }^{22}$ adjusted to a more traditional parameterization of nonlinear wave evolution, as well as the use of a forcing consisting of operational wind analysis from the Integrated Forecasting System of the European Centre for Medium Range Weather Forecasting (ECMWF). Our wave model also uses a daily sea ice concentration map also provided by ECMWF. This global model is discretized with a regular grid in latitude and longitude, using a $0.5^{\circ}$ spacing, and the spectral space is also discretized using 24 directions and 32 frequencies exponentially spaced from 0.037 to $0.72 \mathrm{~Hz}$, such that the relative increase from one frequency to the next is $10 \%$.

For the application off the French coast, we refine the spatial resolution using a triangle-based grid $^{28,29}$ with a varying resolution that increases from $100 \mathrm{~m}$ at the coast to $10 \mathrm{~km}$ at the offshore boundary located at $6^{\circ} \mathrm{W}$, and 47.6 and $48.9^{\circ} \mathrm{N}$. This coastal grid takes into account the same global wind fields; varying water levels and currents are also used, as provided by a depth-integrated flow model. Including these tidal currents is necessary to obtain accurate wave model results in this area. ${ }^{25}$ In the triangle-based grid, wave reflection at the shoreline is parameterized ${ }^{30}$ with a constant beach slope of 0.15 , contrary to a constant energy reflection coefficient $R^{2}=0.1$ for the other domains. This slope typically gives reflection coefficients $R^{2}$ of a few percent only, except for the largest wave periods, say $T>15 \mathrm{~s}$, and the smallest wave heights, of $1 \mathrm{~m}$ or less. ${ }^{30}$ 


\section{ACOUSTIC NOISE IN $100 \mathrm{M}$ DEPTH AND LOCAL SOURCES}

In this section we explore the accuracy of our numerical wave model in terms of second order pressure at very long wavelengths. For this we use pressure measurements that are dominated by A-G modes.

During an experiment in the Iroise Sea off the West coast of France in September 2011, a Seabird SBE-26 tide gauge was deployed on the sea floor in a mean water depth of $110 \mathrm{~m}$, with an acquisition rate of $4 \mathrm{~Hz}$. The raw data were corrected for calibration factors and smoothed to a $2 \mathrm{~Hz}$ sampling. Together with the bottom pressure record, directional wave data were obtained from two Datawell Mark III buoys, one within $300 \mathrm{~m}$ of the tide gauge (SBE26), and the other, buoy number 62069, $25 \mathrm{~km}$ to the East (see Fig. 4).

The experiment includes both neap and spring tides, with the lowest tidal amplitudes occurring on September 6 [Fig. 5(a)] and large tidal amplitudes on September 13. Wave heights decreased from September 6 to September 10, with a large increase in height on September 11, associated with lower peak frequencies [Figs. 5(b) and 5(c)].

Such wave buoys do not measure the full directional spectrum but only four directional parameters for each frequency, which unfortunately do not include $I(f)$, and thus the directional wave distribution $M(f, \theta)$ is usually estimated from these four parameters using statistical methods. Because we also wish to validate the numerical modeling of the acoustic noise sources, we also estimated the full frequency-directional spectrum $F(f, \theta)$ at the location of the buoy using our numerical wave model.

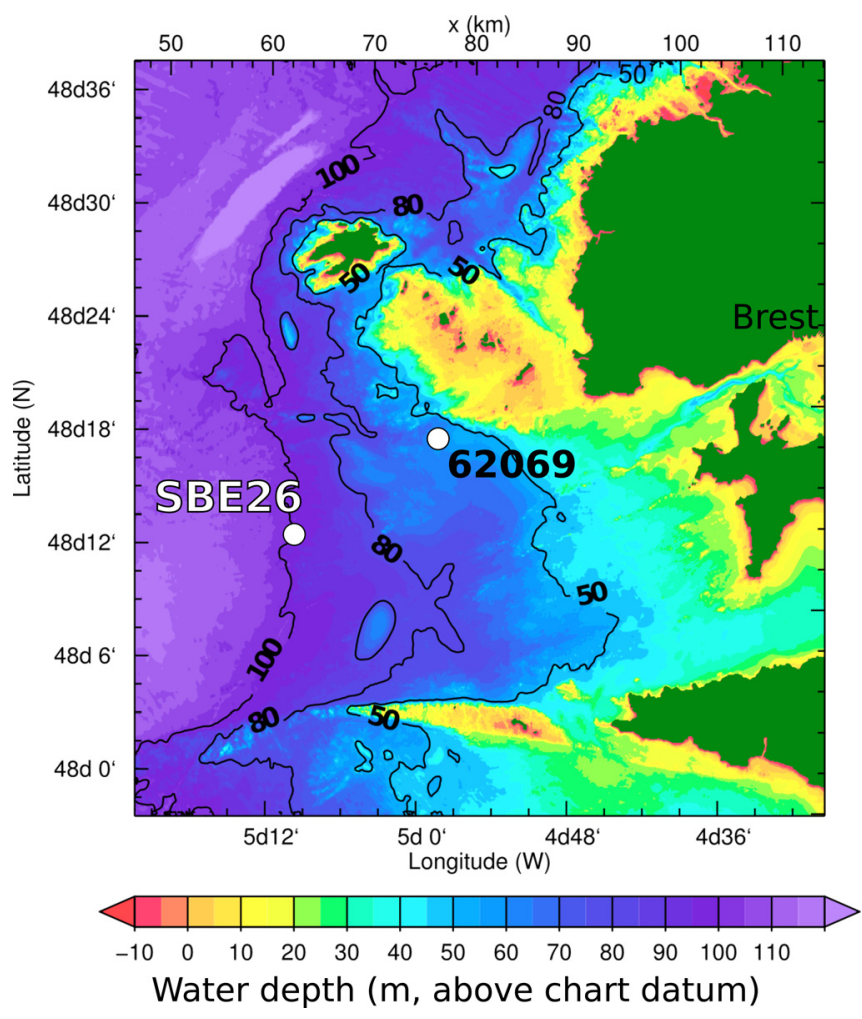

FIG. 4. (Color online) Map of the deployment area in the Iroise sea showing the position of the tide gauge and temporary buoy (SBE26) and the permanent wave buoy (World Meteorological Organization number 62069).
Figure 6(a) shows the frequency spectra recorded on the sea bottom where one clearly distinguishes the infragravity domain (1) for frequencies less than $0.05 \mathrm{~Hz}$, the domain of the dominant wind sea and swell (2), from 0.05 to $0.16 \mathrm{~Hz}$, and the domain of second-order forced signals (3), above $0.16 \mathrm{~Hz}$. As expected from previous studies, ${ }^{28,29}$ the model faithfully reproduces the bottom pressure in domain (2), as shown in Fig. 6(b). We have also verified the good agreement between the modeled spectrum at higher frequency and the measurements of the surface buoy, with a correlation $r=0.94$ and a normalized root mean square error of $15 \%$ for wave height defined over a restricted frequency range from 0.17 to $0.25 \mathrm{~Hz}$. The modeled second order pressure given by Eq. (B1) is shown in Fig. 6(c). The difference between modeled and measured values can be attributed to errors in the estimate of the integral $I(f)$ [Eq. (6)]. We note that the integrals of Eqs. (11) and (B1) have been estimated using the discretized ocean wave spectrum and an analytical integration of coupling coefficient, to avoid a dependance of the solution on the discretization due to the near-singularity of that coefficient for opposing waves.

The difference between panels (a) and (c) in Fig. 6 suggests that, at frequencies below $0.2 \mathrm{~Hz}$, the model probably underestimates $I(f)$ by a factor of 2 to 10 . At higher frequencies, the measurements are closer to the noise floor of the instrument. For visualization in Fig. 7, we have integrated the noise level over the acoustic frequency band 0.2 to $0.5 \mathrm{~Hz}$ (ocean wave frequencies 0.1 to $0.25 \mathrm{~Hz}$ ). This recorded pressure amplitude is generally consistent, but $20 \%$ to $30 \%$ larger than the modeled amplitude. Because the parameterization of shoreline reflection is not very accurate, we have verified that the results are similar when setting the energy reflection coefficient $R^{2}$ to zero (green line in Fig. 7).

The full model based on Eq. (B1) is itself very close to the deep water approximation given by Eq. (11), provided that the bottom pressure amplitude at $z=-h$ is multiplied by 2. This factor of 2 corresponds to the finite depth effect, because $\cosh (k h) \simeq \exp (k h) / 2$ for $k h \gg 1$. However, the simplified expression in Eq. (11) cannot reproduce correctly the energy for $f_{s}<0.2 \mathrm{~Hz}$. For example, if we wish to have $\exp (K z)<0.1$ and $K<0.3 k$, this is only valid for $f_{s}>0.26 \mathrm{~Hz}$.

Equation (B1) provides acceptable estimates of the observed spectral levels for $f_{s}>0.2 \mathrm{~Hz}$, which corresponds to wave frequencies higher than the dominant wave frequency. The difference between the black and blue curves in Fig. 7 can be attributed to errors in the modeled integral $I(f)$, associated to poor shapes of the directional wave spectra coming out of the wave model. Indeed, Herbers and Guza ${ }^{31}$ have shown with measured wave spectra that Eq. (B1) is well verified. Hence, $I(f)$ from our modeled spectra is lower by $20 \%$ to $50 \%$ compared to real spectra, but the variability of the noise level is still well captured. In particular, Fig. 7 shows strong oscillations of the noise level with the semidiurnal tidal period $(12.4 \mathrm{~h}$ ), on September 13 and 14. The noise level at $0.3 \mathrm{~Hz}$ has a pronounced maximum $1 \mathrm{~h}$ after low tide, whereas the OSGW spectrum $E(f)$ hardly changes, which means that the change must come from $I(f)$. The model also predicts a doubling of $I(f)$ that yields a 

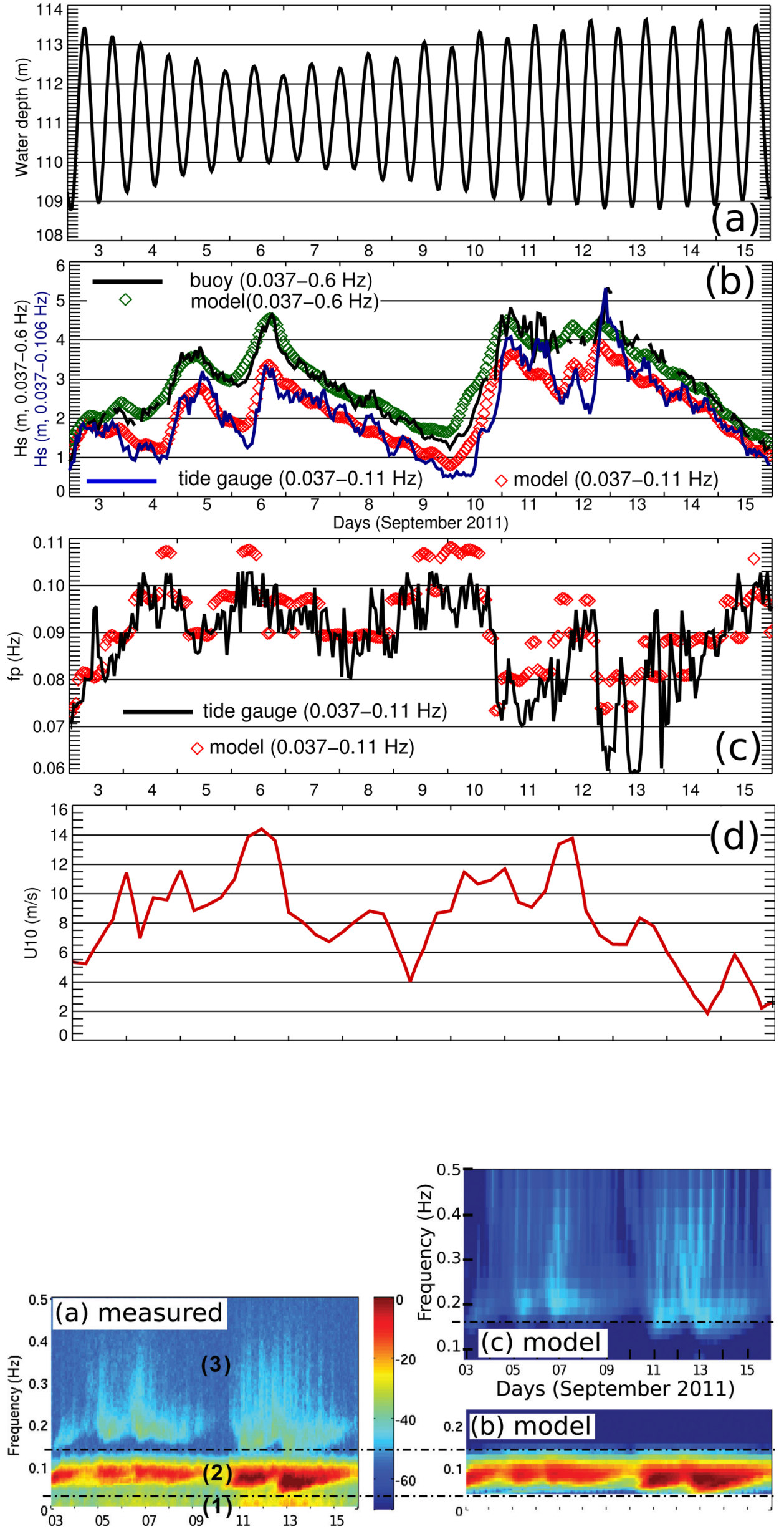

FIG. 5. (Color online) Wave and weather conditions during the experiment. (a) Observed tide, (b) and (c) observed and modeled wave conditions, and (d) modeled wind speed during the FROMVAR 2011 experiment. The modeled wave heights are compared to those estimated from the tide gauge up to $0.106 \mathrm{~Hz}$ - assuming zero current - and those recorded by the wave buoy, over the full buoy frequency range, up to $0.6 \mathrm{~Hz}$.
FIG. 6. Spectra of pressure in $100 \mathrm{~m}$ depth from September 3-16, 2011, from (a) measurements, (b) modeled first order spectrum using linear wave theory, and (c) second order spectrum obtained with the full integral [Eq. (B1)] at lower frequency. The spectral levels are in $\mathrm{dB}$ relative to $1 \mathrm{~Pa}^{2} / \mathrm{Hz}$, ranging from -55 to 0 in the case of the measurements. Data comes from the SBE26 tide gauge with a location indicated in Fig. 4. 

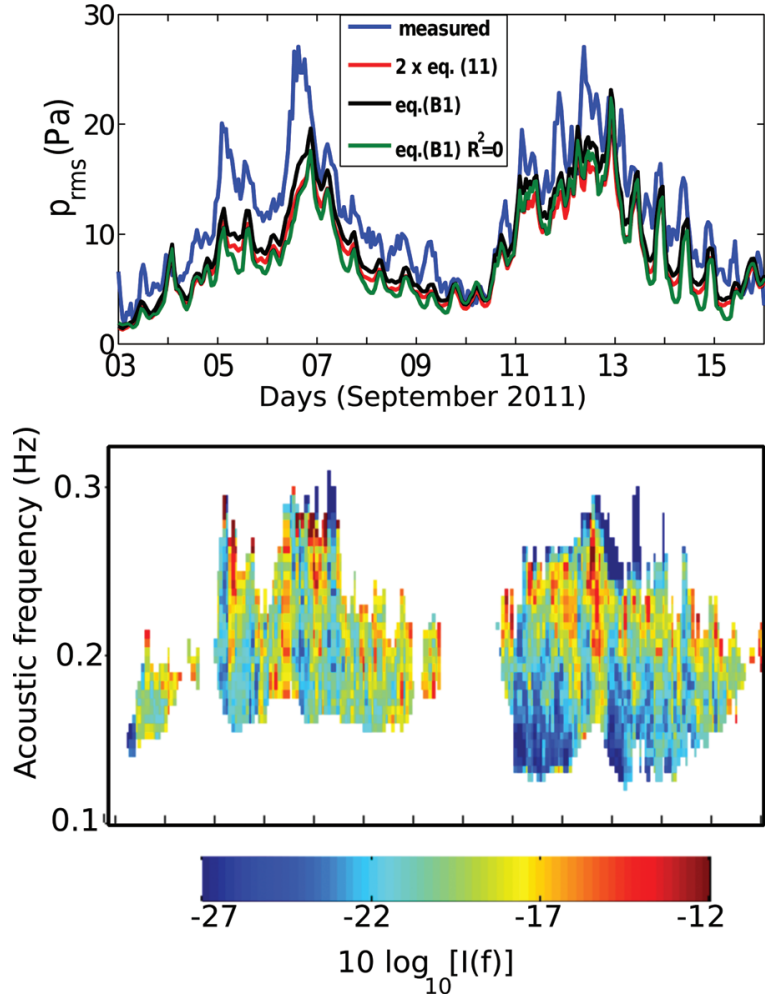

FIG. 7. (a) Standard deviation of bottom pressure in the frequency band 0.2 to $0.5 \mathrm{~Hz}$. The observed pressure is estimated by summing the recorded spectrum after removing the expected measurement noise floor of $3 \times 10^{2}$ $\mathrm{Pa}^{2} / \mathrm{Hz}$ from the power spectrum. A different pressure estimate using the same model without coastal reflection $R^{2}$ is also shown with the green line. (b) Value of the integral $I(f)$ estimated from the recorded noise level and modeled wave spectrum $E(f)$, using Eq. (B1).

modulation of the predicted noise level that agrees well with the observations. This increase of $I(f)$ in the model is related to wave refraction by the tidal currents. ${ }^{28,29}$ The tidal modulation of the predicted noise level disappears if currents are not taken into account, whether or not shoreline reflection is included in the model.

At lower frequencies, below the peak of the surface elevation spectrum for $0.16<f_{2}<0.2 \mathrm{~Hz}$, the bottom pressure amplitude is underestimated by a factor of 2 . The surface buoy measurements also show a directional spreading below the peak that is larger than the model used here. That effect is probably partly due to nonlinear wave-wave interactions that contribute to the buoy measurement, ${ }^{32}$ but the second order pressure data suggest that the model probably has errors at wave frequencies below the peak. These errors may be a stronger than expected coastal reflection, or a directional broadening due to other effects, possibly a poorly approximated non-linear energy flux.

When comparing the propagating and evanescent contributions to pressure, given by Eqs. (9) and (11) it appears clearly that the A-G evanescent modes dominate for water depths less than $z_{a g}=c_{w} /\left(f_{s} \pi \sqrt{8}\right) \simeq c_{w} /\left(9 f_{s}\right)$, while the acoustic modes should dominate farther down. For an acoustic frequency of $0.25 \mathrm{~Hz}$, this depth is $z_{a g}=600 \mathrm{~m}$. We shall now see that the bottom plays a prominent role for acoustic modes, and will make this transition depth $z_{a g}$ shallower.

\section{ACOUSTIC NOISE AT THE ALOHA CABLED OBSERVATORY (ACO)}

The acoustic data used here were measured by a broadband hydrophone (AOS model E-2PD, Atmosphere Ocean Science Inc.) at the ACO Proof Module located $100 \mathrm{~km}$ north of Oahu, HI $\left(22^{\circ} 44.324^{\prime} \mathrm{N}, 158^{\circ} 0.372^{\prime} \mathrm{W}\right)$. This hydrophone, floating $10 \mathrm{~m}$ above the seabed in $4720 \mathrm{~m}$ of water, recorded continuously from February 16, 2007 to October 22, 2008.

\section{A. Average noise at dominant frequencies, 0.1 to $0.3 \mathrm{~Hz}$}

A detailed analysis ${ }^{17}$ of the data showed that spectral densities were highly correlated with the Kipapa (KIP) seismic station, located $120 \mathrm{~km}$ from ACO, on the island of Oahu $(r>0.9$ over the frequencies 0.1 to $0.5 \mathrm{~Hz}$ ). Correlation with other seismic stations varies from $r>0.85$ with the Pohakuloa (POHA) seismic station on Big Island (Hawaii), to $r>0.7$ (only for frequencies between 0.1 and $0.2 \mathrm{~Hz}$ ) with North American stations in Corvallis (Oregon) or Kodiak Island (Alaska). This important correlation throughout the Pacific does not necessarily mean that the sources of the noise recorded at ACO are the same as those of noise recorded at other seismic stations: For many events several sources appear almost at the same time but in different locations. However, with stations KIP and POHA, the correlation is very high and the numerical noise model supports the conclusion that the noise sources are indeed the same for these instruments located only a few hundred kilometers apart.

The time variation of the noise field at KIP was shown to be very well explained by the wave-wave interaction theory of Longuet-Higgins and Hasselmann, ${ }^{7,8}$ at least for frequencies up to $0.3 \mathrm{~Hz}$, with noise levels consistent with expected seismic wave attenuation. ${ }^{19}$ Root-mean-square (rms) ground motions at KIP were modeled using that theory applied to numerical wave model results, and a model-data correlation of 0.86 for 3 -hourly values was obtained. ${ }^{10}$ In that model, most noise sources are in deep water, within $2000 \mathrm{~km}$ from Oahu.

Here we model the acoustic noise at ACO using the method previously used for seismic noise, ${ }^{10}$ except that now the Rayleigh wave energy is propagated separately for the different modes and finally converted to pressure using Eq. (16). However, we still use the same quality factor $Q$ for the attenuation of all these modes and a constant group velocity $U=1.8 \mathrm{~km} / \mathrm{s}$ in Eq. (7).

The analysis of seismic noise at KIP shows that the $Q$ factor is probably on the order of 600 to 1000 , based on model-data correlations, ${ }^{10,25}$ and consistent with the higher $Q$ values expected for older crust ${ }^{33}$ and inferred from acoustic noise in the Pacific. ${ }^{19}$ A constant $Q=800$ corresponds to an attenuation factor $\alpha$ varying from $4.3 \times 10^{-4} \mathrm{~km}^{-1}$ at $f_{s}=0.1 \mathrm{~Hz}$ to $1.3 \times 10^{-3} \mathrm{~km}^{-1}$ at $f_{s}=0.3 \mathrm{~Hz}$. With these values, the shape of the acoustic spectrum is relatively well reproduced (Fig. 8), although the noise level at $1.5 \mathrm{~Hz}$ is overestimated by $5 \mathrm{~dB}$. In practice, we have thus used a stronger attenuation for high frequencies, taking the form $Q=320\left[1+\left(1-\tanh \left(15\left(f_{s} / 1 \mathrm{~Hz}-0.14\right)\right)\right)\right]$ that decreases from $Q=800$ for $f_{s}=0.1 \mathrm{~Hz}$ to 320 at high frequencies. The 

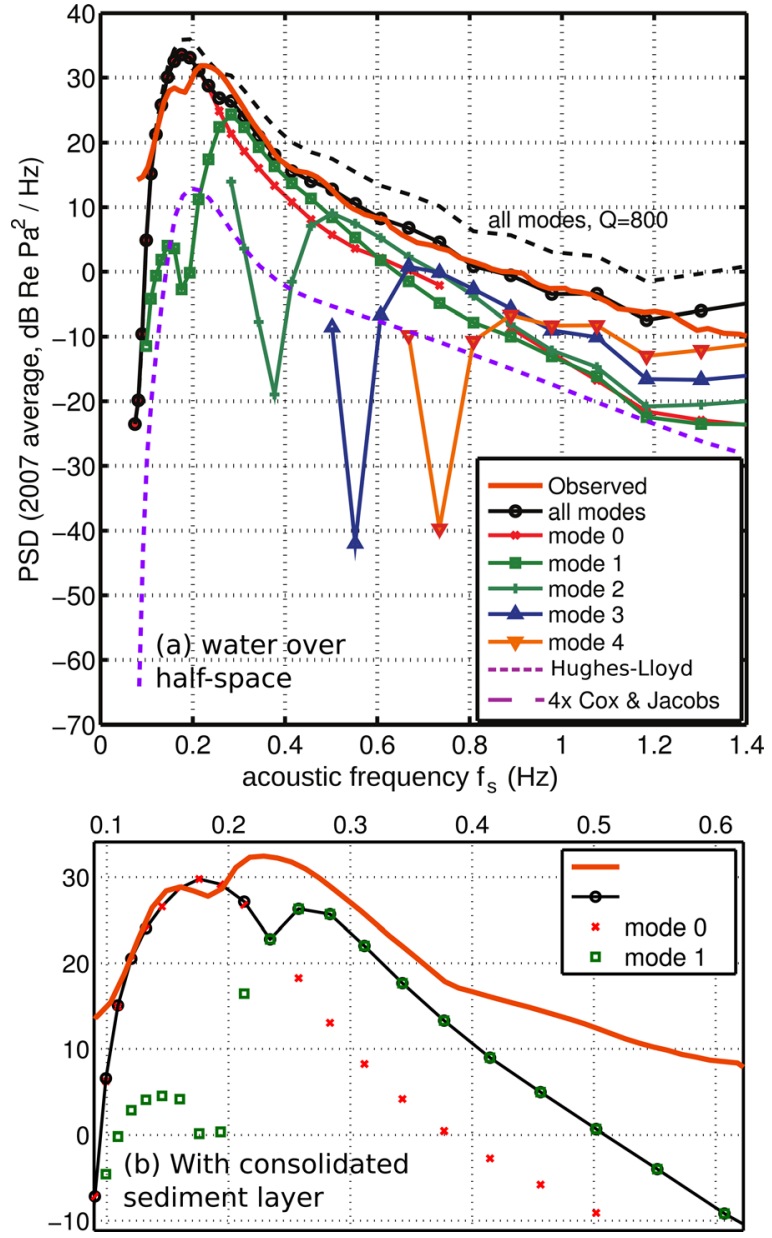

FIG. 8. (Color online) Mean pressure spectrum over the year 2007 at the ALOHA cabled observatory. Measurements are compared to a numerical model of the noise for Rayleigh waves or using the unbounded ocean theory of Lloyd (Ref. 14) or the A-G modes, amplified by a factor of 4 to account for near-bottom effects. The Rayleigh wave propagation is computed using a frequency-varying damping coefficient $Q=320\left[1+\left(1-\tanh \left(15\left(f_{s} / 1 \mathrm{~Hz}-\right.\right.\right.\right.$ $0.14)$ ))], or a constant $Q=800$. In (a) the bottom is modeled like a homogeneous half space, with a response shown in Fig. 2(a). In (b) the bottom is modified with the addition of one layer, in order to mimic the HILO31 model of Abramovici (Ref. 34).

spatial attenuation coefficient is thus increased to $\alpha=2.5 \times 10^{-3} \mathrm{~km}^{-1}$ for $f_{s}=0.3 \mathrm{~Hz}$. This adjusted attenuation gives a good match with the observed seismic noise on land, ${ }^{10}$ as well as the recorded acoustic spectrum, except between 0.16 and $0.28 \mathrm{~Hz}$.

Following the early analysis of bottom effects on acoustic noise by Abramovici ${ }^{34}$ we have tested the effect of different bottom properties by using the first two layers of his HILO31 model: This means a water layer of $4390 \mathrm{~m}$ over a layer of thickness $1 \mathrm{~km}$ with velocities $c_{s s}=1.57$ and $c_{s p}=4.2 \mathrm{~km} / \mathrm{s}$, and a density of $\rho_{s}=1400 \mathrm{~kg} / \mathrm{m}^{3}$, over a half-space of density $\rho_{c}=2.4 \mathrm{~kg} / \mathrm{m}^{3}$ and velocities $c_{c s}=2.94$ and $c_{c p}=6.06 \mathrm{~km} / \mathrm{s}$. As already shown, ${ }^{34}$ these velocities tend to shift the peak of the waveguide response to lower frequencies. If we further take into account the fact that mode 0 has lower group speeds than mode 1 (typically 1.2 and $2.4 \mathrm{~km} / \mathrm{s}$ for depths around $5000 \mathrm{~m}$ and seismic frequencies around $0.2 \mathrm{~s}$ ), then the observed dip in the spectrum around $0.19 \mathrm{~Hz}$ is better reproduced by the model, as shown in Fig. 8(b). Evidence for these two peaks was also found by Bradner et al., in the coherence between mid-water and bottom measurements. ${ }^{35}$ It thus appears plausible that the dip at $0.18 \mathrm{~Hz}$ in the ACO data is not an artifact due to the instrumental response as initially proposed ${ }^{17}$ but rather is a real feature of the noise field, associated with the transition from a dominant mode 0 to a dominant mode 1 Rayleigh wave. This conclusion could be confirmed by the direct observation of the vertical mode structures, using a vertical array of hydrophones.

At this very large depth, the contribution of evanescent modes $^{2}$ is expected at $40 \mathrm{~dB}$ or so below the measured values. We also note that the modeled noise level is $15 \mathrm{~dB}$ above what is expected from the Hughes-Lloyd theory, using the same wave spectra over a bottomless ocean. If we attempt to estimate $I(f)$ from the modeled value of $E(f)$ and the Hughes-Lloyd theory, we would obtain $I(f) \simeq-2 \mathrm{~dB}$ at $f_{s}=1 \mathrm{~Hz}$. This differs from the previous similar estimate by Duennebier et al. ${ }^{17}$ who found $I(f) \simeq-10 \mathrm{~dB}$, assuming a Pierson-Moskowitz shape in frequency. ${ }^{36}$ The PiersonMoskowitz shape is not the problem here, as shown by Fig. 9(a). Instead, their use of the Hughes-Lloyd theory was not completely consistent and produced an overestimation of the noise level by $8 \mathrm{~dB}$. In particular their Fig. 8 shows a noise level near $0 \mathrm{~dB}$ re $1 \mathrm{~Pa}^{2} / \mathrm{Hz}$ for $f_{s}=1 \mathrm{~Hz}$ when
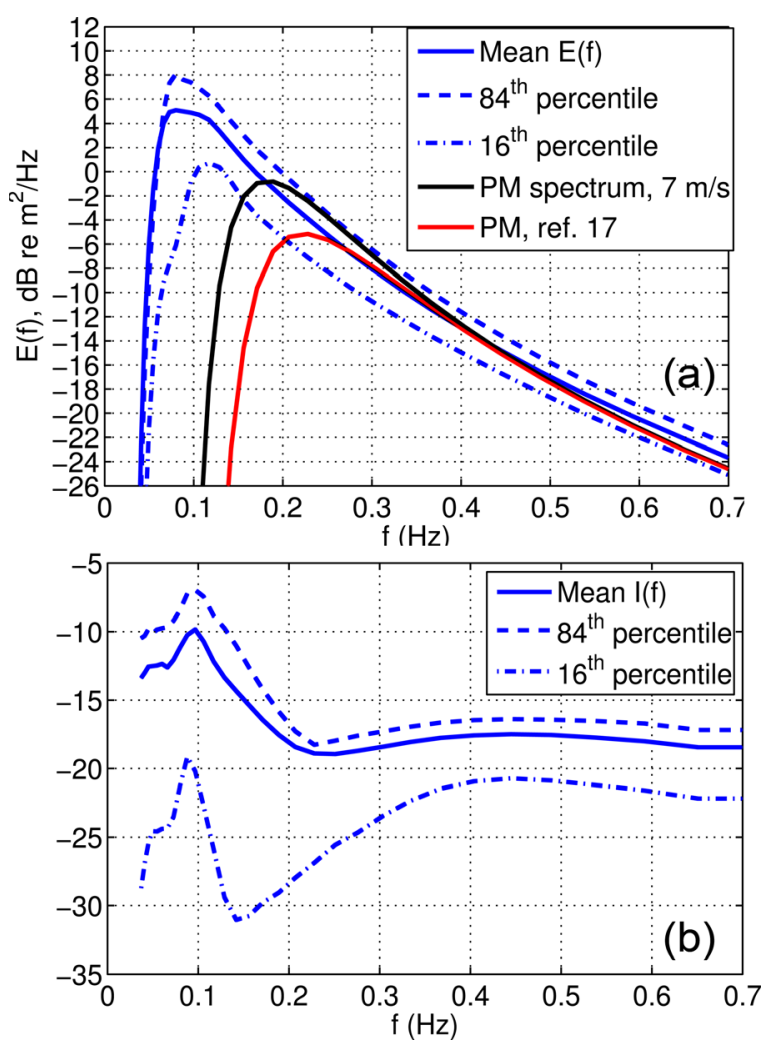

FIG. 9. (Color online) Mean value and typical ranges for (a) the wave energy spectrum and (b) the overlap integral estimated at the location of ACO using our numerical wave model. The 16th and 84th percentiles would correspond to minus and plus one standard deviation if the distribution were Gaussian. For reference, a Pierson-Moskowitz spectrum with a wind speed $U_{10}=7 \mathrm{~m} / \mathrm{s}$ is also shown. We also note that in Ref. 17 the peak frequency was estimated using $f_{p}=\nu_{\mathrm{PM}} g / U_{10}$ with $\nu_{\mathrm{PM}}=1.56 \mathrm{~m}^{2} \mathrm{~s}^{-2}$ instead of the more standard value $\nu_{\mathrm{PM}}=1.28 \mathrm{~m}^{2} \mathrm{~s}^{-2}$, giving the spectrum shown in black. Both are consistent with the original data (Ref. 36). 
using $I(f)=-8 \mathrm{~dB}$, whereas previous results ${ }^{24}$ give for that frequency a noise level of $-7.6 \mathrm{~dB}$. However, with $-2.3 \mathrm{~dB}$ at $f_{s}=1 \mathrm{~Hz}$, the average noise level at ACO is higher, suggesting that the Hughes-Lloyd theory is not sufficient to explain the observed noise. Indeed, consistent with the analysis by Webb for other sites ${ }^{19}$ this noise level agrees with Rayleigh wave theory. ${ }^{8}$

The analysis in Ref. 17 of the relative change of $I(f)$ with wind speed is still relevant, only the absolute value of $I(f)$ is not correct because bottom effects have been neglected. Even here with a reasonable estimate of bottom effects, the uncertainty on the seismic attenuation factor $Q$ and the shape of seismo-acoustic modes make the estimate of $I(f)$ from noise very difficult.

With our previous analysis of shallow water data, we have no validation of the wave model in terms of $I(f)$ for frequencies higher than $f_{s}=0.4 \mathrm{~Hz}$, and the agreement here of model and data can be the result of the adjustment of the seismic attenuation $Q$. As expressed by Eq. (18) a factor of 2 error on $I(f)$ can be compensated by a factor of 0.5 error on $Q$ or on the group speed of Rayleigh modes. Although we can use land-based seismic data to constrain $Q$ at low frequencies, ${ }^{10}$ there is no such constraint for the higher frequencies.

\section{B. Noise variability}

The temporal variability of the noise level is very well captured by our model, with a correlation $r=0.86$ between the measured and modeled 3-hourly time series of the rms pressure (Fig. 10). This correlation only shows that the noise model variability is realistic for the dominant noise components, at frequencies $f_{s}$ around $0.2 \mathrm{~Hz}$. This dominant noise is not caused by coastal reflection (class II events), because the same model without reflection gives noise levels for $f_{s}>0.15 \mathrm{~Hz}$ that are changed by less than $1 \mathrm{~dB}$. Instead, this noise is mostly caused by the interaction of different wave systems (class III events), as illustrated in Ref. 10. Toward

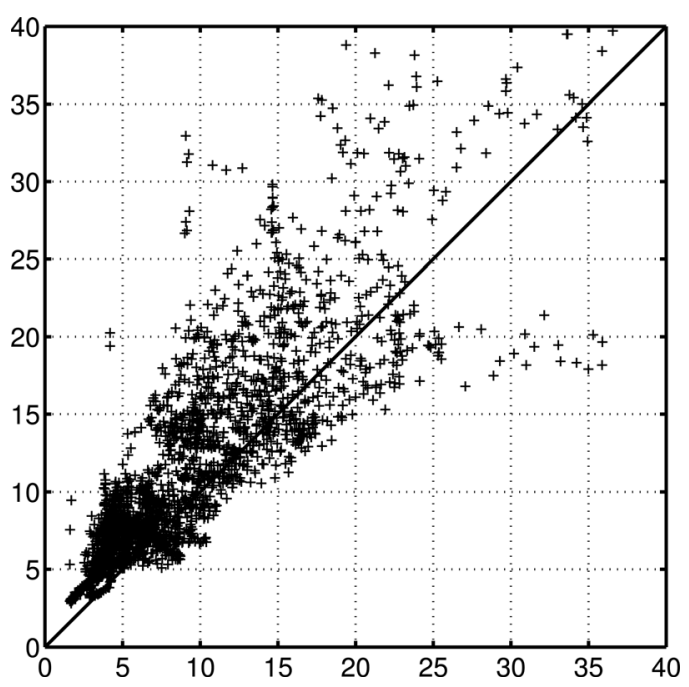

FIG. 10. RMS pressure in the frequency band 0.07 to $1.4 \mathrm{~Hz}$ at ACO. The same figure is obtained if the frequency range is 0.15 to $0.3 \mathrm{~Hz}$. Each symbol corresponds to a $3 \mathrm{~h}$ record. The data used here spans February 26 to December 31, 2007. higher frequencies, that correlation degrades, as indicated in Table I.

\section{Interpretation of noise for frequencies 0.3 to $1 \mathrm{~Hz}$ in terms of wave directionality}

Noise in the frequency range 0.3 to $1 \mathrm{~Hz}$ is expected to come mostly from class I events, corresponding to the waves generated by the local wind, ${ }^{17}$ for which wave directional properties are generally poorly known. ${ }^{19}$

From Hasselmann's theory, the noise sources are proportional to the product $I(f) E^{2}(f)$ at $f=f_{s} / 2$ [see Eq. (7)], where $E(f)$ is the direction-integrated spectral density of the surface elevation associated with gravity waves, and $I(f)$ is an integral of the directional wave spectrum that is non-zero if and only if there are wave trains of frequency $f$ that propagate in opposite directions. Our results in Sec. IV are one of the few datasets from which $I(f)$ can be estimated, up to $0.4 \mathrm{~Hz}$. Off the French coast, our model estimates of $I(f)$ are consistent within a factor of $4(6 \mathrm{~dB})$ with the measured pressure power. At ACO, our model predicts that $I(f)$ varies from -28 to $-15 \mathrm{~dB}$ for $f=0.5 \mathrm{~Hz}$. Only near-surface pressure measurements ${ }^{31}$ or other types of measurements, such as stereo-video imagery ${ }^{37}$ may provide a strong test of our model for $I(f)$. No such open ocean data has yet been analyzed for $f_{s}>0.4 \mathrm{~Hz}$.

The other poorly constrained process in our noise model is the attenuation, represented by $Q$ or $\alpha=2 \pi f_{s} /(U Q)$. However, assuming that propagation effects (refraction and attenuation) are linear, we expect that the noise power is a linear function of the source power, so that the recorded variability of the sound power spectral density (PSD) at a fixed frequency $f_{s}$ should be proportional to the variability of the spectral density of noise, i.e., $I(f) E(f)^{2}$ at $f=f_{s} / 2$. Hence, if $E(f)$ is well modeled and if the noise theory is right, we can estimate the relative variations of $I(f)$ from the noise records.

TABLE I. Correlation coefficients for the noise spectral level at ACO with various wave and wind-related parameters, and for acoustic frequencies $f_{s}$ ranging from 0.3 to $1 \mathrm{~Hz} . U_{10}$ is the wind speed at $10-\mathrm{m}$ height given by operational ECMWF analyses. The other wave parameters $E(f)$ and $I(f)$, with $f=f_{s} / 2$, are estimated from the model spectral output at ACO, while the "noise model" uses the Rayleigh wave propagation model described above, and thus integrates the noise sources, proportional to $I(f) E(f)^{2}$, over the entire ocean. Noise can be correlated to $E^{p}(f)$ or $U_{10}^{q}$. The exponents $p$ and $q$ that give the best least squares fit are listed in the bottom line. For $f_{s}=1 \mathrm{~Hz}$ we also give results with the BJA (Ref. 38) and TC (Ref. 39) wave model parameterizations.

\begin{tabular}{lccrcc}
\hline \hline Parameter & & \multicolumn{5}{c}{$1.0 \mathrm{~Hz}}$, \\
\hline Noise model & 0.82 & 0.76 & 0.52 & & \\
Wind speed $U_{10}$ & 0.09 & 0.46 & 0.73 & 0.73 & 0.73 \\
$E^{2}(f)$ & 0.27 & 0.60 & 0.76 & 0.51 & 0.34 \\
$I(f)$ & 0.49 & 0.16 & -0.25 & -0.13 & -0.09 \\
$I(f) E^{2}(f)$ & 0.74 & 0.70 & 0.37 & 0.05 & 0.05 \\
Best fit exponents & $0.38(1.5)$ & $1.4(2.3)$ & $2.3(2.5)$ & 1.6 & 1.7 \\
$p(q)$ & & & & & \\
\hline \hline
\end{tabular}


Although we do not have measurements of $E(f)$ right around ACO, we can compare the wave model with buoy data from around Hawaii. Buoy number 51001 is a 3-m discus-shaped buoy located $330 \mathrm{~km}$ north-west of Oahu, that should properly resolve waves up to a frequency of $0.4 \mathrm{~Hz}$. Compared to these data, our model results have a rms error of $20 \%$ for $E(f)$ at $f=0.4 \mathrm{~Hz}$, which includes a $7 \%$ positive bias, and correlation $r=0.88$.

In order to illustrate these uncertainties we evaluate the capabilities of our noise model and also two alternative models obtained by replacing our parameterization for spectral wave evolution, ${ }^{22}$ i.e., how fast the various wave components grow under wind forcing and decay due to wave breaking. We have used the parameterizations by Bidlot, Janssen, and Abdallah (hereinafter BJA) used at the European Center for Medium Range Weather Forecasting, ${ }^{38}$ or the ones by Tolman and Chalikov ${ }^{39}$ (hereinafter TC), used until May 2012 at the U.S. National Center for Environmental Prediction (NOAA/NCEP) and used in the seismic noise model by Kedar et al..$^{9}$ Although these two parameterizations give larger errors than ours for a wide range of wave parameters, ${ }^{22}$ their possible validity for $I(f)$ has not been thoroughly tested. The BJA parameterization yields $r=0.78$ with an rms error of $18 \%$ and $5 \%$ negative bias for $E(f=0.4 \mathrm{~Hz})$ at the buoy 51001, and the TC parameterization gives $r=0.81$, a $9 \%$ positive bias but a rms error of only $16 \%$. All these errors are low enough so that we expect that the relative changes in $I(f)$ can be obtained from the measured noise level and the modeled $E(f)$.

The noise model integrates over the ocean surface the source $S_{\mathrm{DF}}$ that is proportional to $E^{2}(f) I(f)$. The mean modeled noise level can be adjusted by changing the attenuation factor $Q$, as shown in Fig. 8, which gives more or less weight to the remote sources. From seismic noise studies, we know that the relevant sources of noise at the dominant acoustic frequency of $0.2 \mathrm{~Hz}$ are expected to be mostly within a few hundred kilometers from the hydrophone, while significant events at lower frequencies can be several thousand kilometers away, ${ }^{10}$ and these modeled source regions have typical correlation distances of hundreds of kilometers, except for the relatively small part that is associated to shoreline reflection.

For the highest frequencies the remote sources become less important and the modeled noise level becomes proportional to the local value of $E^{2}(f) I(f)$, as given by Eq. (17). The correlation between the local value of $E^{2}(f) I(f)$, and our full noise model increases from $r=0.54$ at $f_{s}=0.13 \mathrm{~Hz}$, to $r=0.86$ at $f_{s}=0.15 \mathrm{~Hz}$, and reaches $r=0.96$ for $f_{s}=0.8 \mathrm{~Hz}$, which gives an idea of the diminishing importance of the noise sources located in regions beyond the spatial correlation distance of the modeled wave field, as expected by Eq. (17). For $f_{s}$ above $0.8 \mathrm{~Hz}$, the measured noise level correlates better with the local wind speed, as found in previous studies, ${ }^{17}$ or even better with the wave spectral density $E(f)$. Table I summarizes the correlations between recorded noise spectral densities and various modeled wind or OSGWrelated parameters.

This correlation is also illustrated by Fig. 11. The transition from a good fit at $0.3 \mathrm{~Hz}$ [Fig. 11(a)] to a low bias at
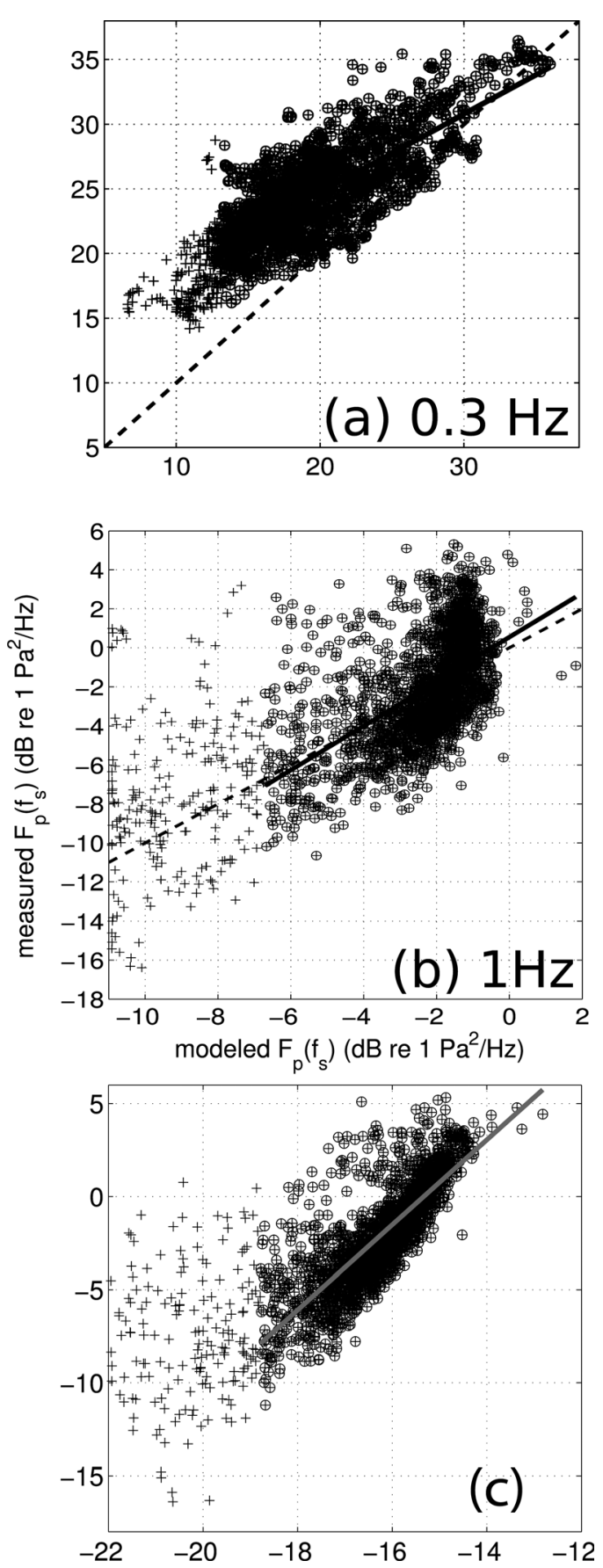

FIG. 11. Modeled versus observed spectral densities. (a) $f_{s}=0.3 \mathrm{~Hz}$ and (b) $f_{s}=1 \mathrm{~Hz}$. At this higher frequency, the observed noise level correlates better with the wind speed or (c) with the modeled wave spectral density $E(f)$. Data points without circles correspond to the lowest $10 \%$ in the modeled values, which were excluded when computing the best fit exponents. The dashed line indicates the 1 to 1 correspondence. The solid lines indicate the best fit between model and observations.

$1 \mathrm{~Hz}$ [Fig. 11(b)] is probably the result of both a directional spectrum that is too narrow and an underestimation of the seismic attenuation.

In general, the decrease in correlation for $f_{s}>0.6 \mathrm{~Hz}$ can be interpreted as a poorly modeled directional spectrum, giving errors on the variability of $I(f)$. Although multiplication of $E^{2}(f)$ by $I(f)$ clearly improves the correlation for $f_{s}<0.6 \mathrm{~Hz}$, this is not the case for the higher frequencies. 
When using BJA and TC parameterizations ${ }^{38,39}$ for the wave evolution, the correlation of $I(f) E^{2}(f)$ with the measured noise level becomes insignificant.

When correlating the modeled wave spectral density $E^{p}(f)$ with the noise level, the best correlation for $f=0.5 \mathrm{~Hz}$ (i.e., $f_{s}=1 \mathrm{~Hz}$ ) is obtained with a power $p=2.3$ (see Table I). If the model variation is correct, we would thus expect that $I(f)$ should vary like $E^{0.3}(f)$. On the contrary, all the wave model parameterizations that we have tested here give a decreasing $I(f)$ when $E(f)$ increases. These different model settings are unable to describe the variability of $I(f)$ for $f_{s}>0.8 \mathrm{~Hz}$. Besides, for those frequencies, BJA and TC parameterizations produce average noise levels that are, respectively, 10 and $20 \mathrm{~dB}$ lower than our model results.

Figure 12 illustrates a typical event with a $10 \mathrm{~dB}$ drop in noise level associated to a drop in wind speed, from 6 to $2 \mathrm{~m} / \mathrm{s}$, and it also shows the fluctuation of noise levels as the winds oscillate around $7 \mathrm{~m} / \mathrm{s}$ the following days. The noise level drop is similar to the busts analyzed by Farrell and Munk $^{24}$ which were attributed to a drop in $I(f)$. Here this event is, according to the model, rather associated to a drop in wave energy $E(f)$. In this respect the model is consistent with an extrapolation of the Moskowitz observations to low winds. With a wind speed of $3 \mathrm{~m} / \mathrm{s}$ on August 24-25, which is also the value reported by the nearby WHOI-Hawaii Ocean Time series Station buoy, the peak frequency of the wind sea should be at $f=0.43 \mathrm{~Hz}$. As a result the wave energy at $0.5 \mathrm{~Hz}$ is already reduced by the low-frequency roll-off in the

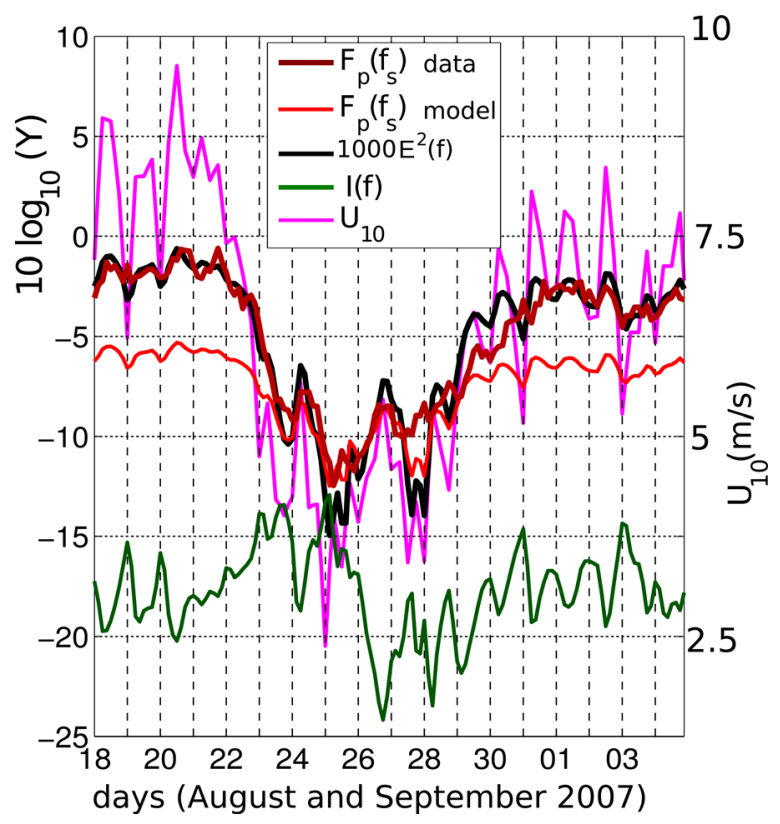

FIG. 12. (Color online) Example of relative variations of various modeled wave-related quantities at a frequency $f=0.5 \mathrm{~Hz}$, the near-bottom pressure PSD at $f_{s}=1 \mathrm{~Hz}$ and the wind speed, at the location of the ALOHA Cabled Observatory. The wind speed is taken from ECMWF 6-hourly analyses. All data are averaged or interpolated at $3 \mathrm{~h}$ intervals. For all quantities except the wind speed, the logarithm is shown: The integrals $I(f)$ that are nondimensional, the wave PSD is expressed in meter squared per Hertz, and the pressure PSD is in $\mathrm{Pa}^{2} / \mathrm{Hz}$. spectrum [see, e.g., Fig. 9(a)]. A similar event at buoy 51001 , on which both winds and waves are recorded, gives a similar pattern on June 13, 2007, when the wind dropped to $4 \mathrm{~m} / \mathrm{s}$ and the spectral density at $0.4 \mathrm{~Hz}$ drops by $6 \mathrm{~dB}$ and nearly $10 \mathrm{~dB}$ at $0.3 \mathrm{~Hz}$.

Instead, when the wind speed is higher, as on September 1 to 5 , the noise level is fairly constant but the model predicts a reduction in $I(f)$ by $3 \mathrm{~dB}$ for a wind increase of $0.8 \mathrm{~m} / \mathrm{s}$, which overwhelms the less than $0.5 \mathrm{~dB}$ increase in $E(f)$.

In summary, the observed noise variability at acoustic frequencies $f_{s}>0.4 \mathrm{~Hz}$ is generally poorly represented by our noise model for wind speeds larger than $6 \mathrm{~m} / \mathrm{s}$ with a strong saturation of the modeled noise at high winds, as shown in Figs. 11(b) and 12. The wave model part could be modified using other proposed parameterizations. The two common alternatives that we have used ${ }^{38,39}$ give worse results, as expected from the better directional wave parameters obtained with our wave model parameterization ${ }^{22}$ compared to these alternatives.

It is thus likely that either the wave model is flawed, and some processes in the wind-wave spectral evolution are poorly represented or missing, or that the noise source and propagation are not well estimated. In particular, wave breaking has been found to generate short gravity waves, an effect that is not taken into account in our wave model, but which was found necessary to reproduce the azimuthal variability of radar observations. ${ }^{17,40}$ This indirect effect of breaking may be an important process for the noise levels at acoustic frequencies above $0.6 \mathrm{~Hz}$.

\section{ACOUSTIC NOISE AT A WEST-KERGUELEN HYDROPHONE (WKER-3)}

\section{A. The dataset and its relation with the ACO data}

In order to further reduce the little known effects of seismic propagation, and test how universal our conclusions may be, we move to a different ocean location, at $46^{\circ} 49^{\prime} \mathrm{S}$ $60^{\circ} 24^{\prime} \mathrm{E}$, west of Kerguelen plateau in the southern Indian ocean, where the water depth is nearly uniform, around $4400 \mathrm{~m}$, over nearly $1000 \mathrm{~km}$. This differs from ACO, which was only $100 \mathrm{~km}$ from islands and submarine ridges. We analyze data recorded from December 2009 to January 2011 with a hydrophone moored in the SOFAR channel $550 \mathrm{~m}$ below the sea surface. At that depth, the effect of the bottom is still very large with an increase by 10 to $20 \mathrm{~dB}$ in acoustic power compared to the bottomless ocean theory [Eq. (10)]. As a result, the contribution of A-G modes is negligible. These data should thus be comparable to the ACO data, except for the generally higher sea states of the roaring 1940 s. A typical $4 \%$ increase in sound velocity between the surface and any part of the water column means that rays leaving the surface at angles less than $16^{\circ}$ from the horizontal will not reach the bottom. However, Rayleigh modes typically correspond to angles between $45^{\circ}$ and $60^{\circ}$, meaning that the SOFAR channel should have little impact on these acoustic components, only shifting slightly the dispersion relation of the Rayleigh modes. 


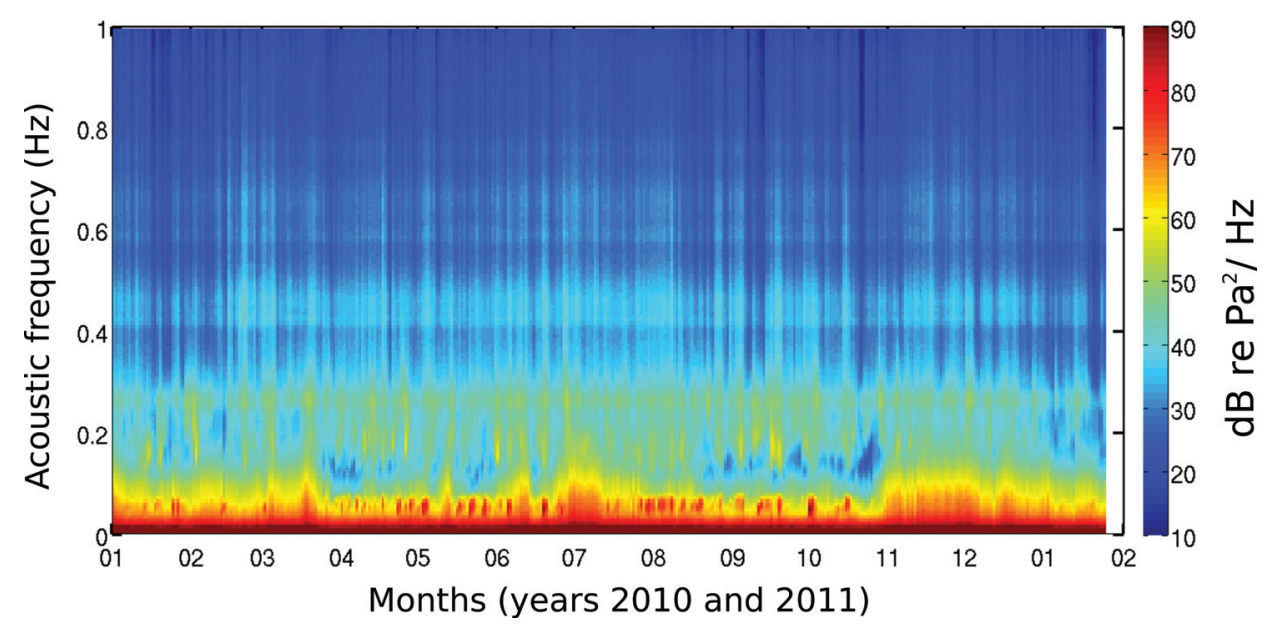

FIG. 13. Spectra of pressure measured by the WKER-3 hydrophone, computed over $160 \mathrm{~s}$ segments and averaged for each day.

The WKER-3 mooring was originally designed to record higher-frequency signals, from large marine mammals and low-energy earthquakes. The sensor (HTI 90-U) has a flat response from $2 \mathrm{~Hz}$ to $2 \mathrm{kHz}$ and an internal highpass filter ramping up $68 \mathrm{~dB}$ from 0.02 to $2 \mathrm{~Hz}$. We have used the calibration curve provided by the manufacturer, without any additional calibration. The actual response of the hydrophone at very low frequencies, below $2 \mathrm{~Hz}$, is thus not well known.

Averaged spectra of pressure power density were computed every $3 \mathrm{~h}$, using windows of $34 \mathrm{~s}$. Thus 340 spectra were averaged for each estimate. We apply here the same modeling technique used in Sec. V for the ACO dataset. We note that the type of analysis performed by Duennebier et al. $^{17}$ for ACO cannot be performed here for frequencies between 5 and $100 \mathrm{~Hz}$ which are dominated by iceberg cracking and marine mammal calls.

The frequency-time spectrogram for the Kerguelen data exhibits vertical and horizontal stripes (Fig. 13) at frequencies below $2 \mathrm{~Hz}$. The vertical bands suggest that the forcing is strongly coherent for all acoustic frequencies higher than $0.2 \mathrm{~Hz}$, which is consistent with the idea that this noise should correspond to wind sea waves with frequencies higher than $0.1 \mathrm{~Hz}$. These are class I noise sources. The variability in the recorded noise level at the dominant frequencies is very well captured by the model, with a correlation coefficient $r=0.86$ between the observed and modeled standard deviation of the pressure over $3 \mathrm{~h}$ intervals, in the frequency band 0.18 to $0.3 \mathrm{~Hz}$. Figure 14 shows that all model results are within a factor of 3 of the observations, with smaller relative errors for the strongest events. This high correlation is comparable to that between observed seismic ground displacement at land stations and model results ${ }^{10}$ based on Eq. (14).

For acoustic frequencies around $1 \mathrm{~Hz}$, we find a significant correlation $(r=0.67)$ with the spectral wave density to a power $1.7, E^{1.7}(f=0.5 \mathrm{~Hz})$, which is similar to what was found for ACO, although the exponent here is somewhat smaller. The correlation with the wind is weaker $(r=0.49)$, and correlation is almost negligible with the local modeled value of $E^{2}(f) I(f)$. That correlation with $E^{2}(f) I(f)$ is $r=0.75$ at $f_{s}=0.3 \mathrm{~Hz}$ but falls to $r=0.40$ at $f_{s}=0.5 \mathrm{~Hz}$. This difficulty of the model to reproduce the higher frequency noise variability thus appears at lower frequencies at WKER-3 compared to ACO. This shift is probably associated with the lower wind sea peak frequencies around Kerguelen compared to Hawaii.

\section{B. Effects of a sediment layer}

The horizontal bands of high energy at constant frequencies (e.g., 0.4 and $0.58 \mathrm{~Hz}$, Fig. 13) are consistent with the presence of acoustic modes in the water column that would correspond to the Rayleigh modes shown in Fig. 2, which were previously recorded in other experiments. ${ }^{19}$ Given the local water depth, the three noise maxima indicated with arrows in Fig. 15 correspond to non-dimensional water depths $f_{s} h / c_{w}$ of $0.75,1.27$, and 1.75 . These are significantly

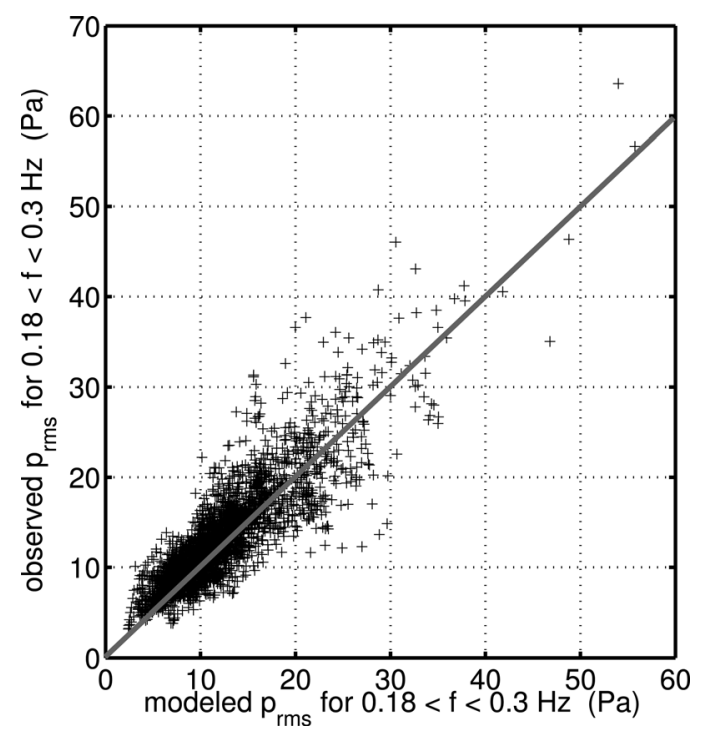

FIG. 14. Three-hourly mean standard deviation of modeled and recorded pressure observed at WKER-3. The noise model combines our numerical wave model that computes the directional wave spectrum $F(f, \theta)$ and its spatial variability over the ocean. This wave spectrum defines the magnitude of the noise source for all ocean locations. These sources are integrated and converted to pressure using Eqs. (14) and (16) with a seismic attenuation factor of $Q=600$ for all modes, which means $\alpha=5 \times 10^{-4} \mathrm{~km}^{-1}$ for $f_{s}=0.1 \mathrm{~Hz}$. 


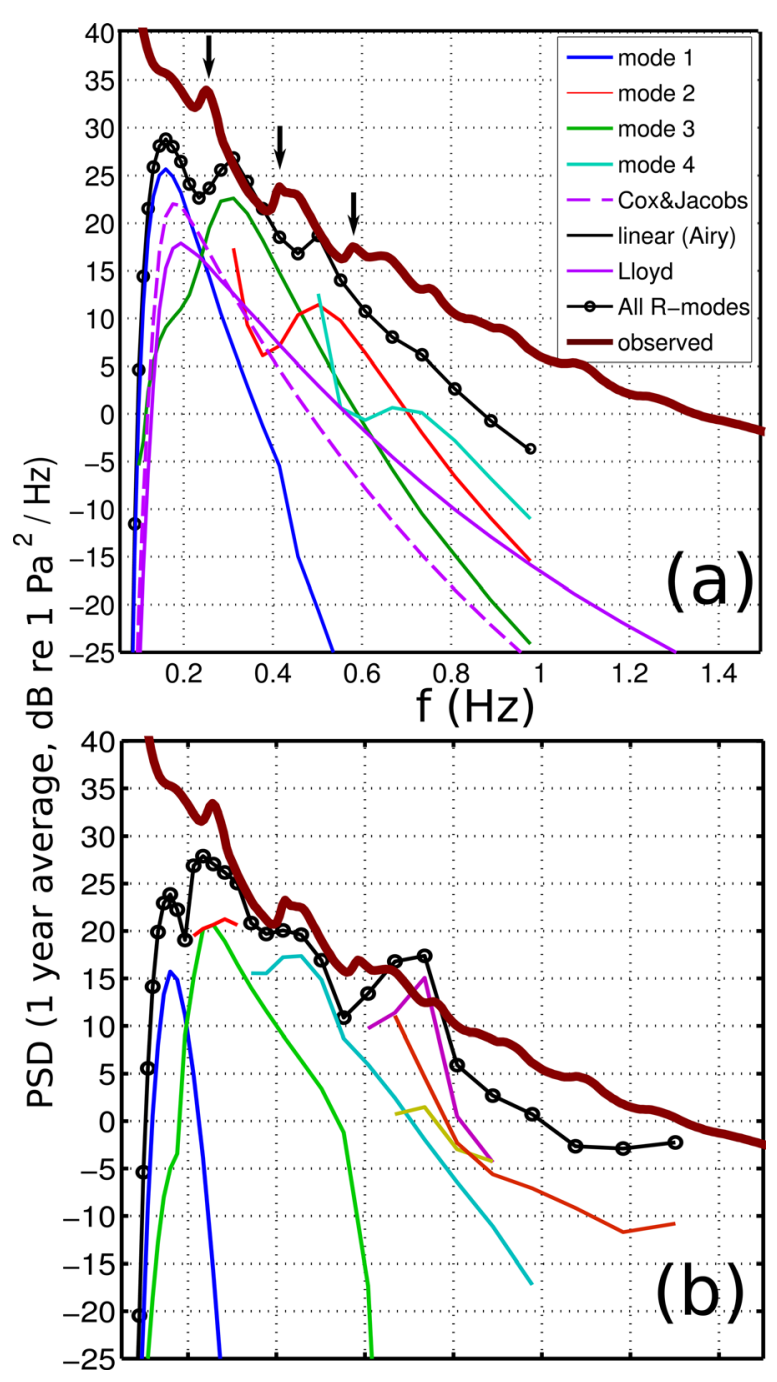

FIG. 15. (Color online) Mean pressure spectrum over the year 2010 at the WKER-3 hydrophone. Units are $\mathrm{dB}$ relative to $1 \mu \mathrm{Pa}^{2} / \mathrm{Hz}$. In (a) the sediment layer is not taken into account, and in (b) a sediment layer of thickness $700 \mathrm{~m}$ in $4400 \mathrm{~m}$ depth is used, corresponding to the amplification coefficients in Fig. 3(b). A constant seismic attenuation $Q=600$ was used for both panels.

lower than the maxima at $0.84,1.41$, and 1.99 expected for a homogeneous crust with the properties used in Fig. 2(a). This shift in the maximum energy is not likely to be caused by the presence of sources in different water depths, as these depths would have to be larger than $5000 \mathrm{~m}$ to explain this shift, and such depths are not found within $2000 \mathrm{~km}$ of the hydrophone.

On the contrary, it is well known that sediment layers may strongly modify the dispersion relations of Rayleigh waves. ${ }^{18}$ This effect has even been used to estimate sediment properties from ambient noise measurements. ${ }^{12,41}$ We have thus explored the possible impact of different bottom layers by adding one homogeneous layer of sediment in the Hasselmann theoretical model (see Appendix A for details). Based on global sediment thickness databases, ${ }^{42}$ the crust between Kerguelen and Crozet islands is covered by at least $500 \mathrm{~m}$ of sediments. We have thus set the sediment layer thickness to $700 \mathrm{~m}$, and from the review by Hamilton ${ }^{43}$ we have chosen a constant $c_{s s}=600 \mathrm{~m} / \mathrm{s}, \rho_{s}=1700 \mathrm{~kg} / \mathrm{m}^{3}$, and $c_{s p}=2 \mathrm{~km} / \mathrm{s}$, as in Figs. 1(b) and 3(b). These geo-acoustic parameters are generally realistic for such a thick layer but we have not attempted to fit the properties of any known sediment type.

This addition of a sediment layer causes a frequency shift of the peaks of maximum noise amplification coefficients $a_{j}$ now appearing at $f_{s} h / c_{w}=0.68,1.35$, and 1.73 , as shown in Fig. 2(b). These peaks agree better with the observed noise maxima [Fig. 15(b)]. The peaks of modes 1 and 2 are slightly shifted toward lower frequencies between Figs. 2(a) and 2(b). New propagation modes also appear [modes 3 and 5 in Figs. 2(b) and 13(b)], which modifies the frequencies of modeled noise peaks. The noise level is also strongly modified, with a $3 \mathrm{~dB}$ amplification of the ground displacement for the mode $j=1$, but a $5 \mathrm{~dB}$ reduction in the associated pressure for $f_{s} \simeq 0.2 \mathrm{~Hz}$, due to a shift of the dispersion relation, which shortens the wavelength and modifies the vertical structure of the mode.

This effect is significant even for much thinner sediment layers. For example, using $c_{s p}=1.8 \mathrm{~km} / \mathrm{s}, c_{s s}=0.3 \mathrm{~km} / \mathrm{s}$, $\rho_{s}=1400 \mathrm{~kg} / \mathrm{m}^{3}$, a layer thickness of $200 \mathrm{~m}$ does not shift the frequencies of the $a_{j}$ maxima but it is enough to reduce the mode 1 bottom pressure noise level by $6 \mathrm{~dB}$, while the mode 2 noise level is increased by $6 \mathrm{~dB}$ due to increase in the amplification coefficient $a_{1}$.

\section{CONCLUSIONS AND PERSPECTIVES}

We have taken advantage of several hydro-acoustic datasets to test a numerical noise model and examine the mode structure of the seismic noise. This noise model is based on a numerical wave model that uses a sequence of modeled wind fields over the ocean, from a numerical weather prediction model, to predict the directional wave spectrum $F(f, \theta)$ which varies in space and time. The other piece of the noise model is a noise source and propagation model based on the theory of Hasselmann, ${ }^{8}$ which says that the local noise source for both evanescent gravity-acoustic modes and propagating seismo-acoustic modes should be proportional to the integral $I(f)$, defined from $F(f, \theta)$ [Eq. (6)], that measures the total amount of wave energy traveling in opposing directions. The propagation in the noise model moves noise energy along great circle routes, with a constant attenuation.

The present model extends the previous work by Webb $^{19}$ in which the shape of $F(f, \theta)$ was parameterized from the local wind. In that approach, part of the variability of the noise source was missed due to the finite time of adjustment of the waves to the wind fields. Confirming previous studies, ${ }^{10,19,30}$ the accuracy of modeled directional distributions of ocean waves is critical to estimate $I(f)$.

By analyzing near-surface pressure measurements, we primarily observed gravity-acoustic waves that are functions of the local sea state only. Our observations from the French Atlantic continental shelf suggest that the model underestimates the noise source by $20 \%$ to $50 \%$, especially for acoustic frequencies below $0.2 \mathrm{~Hz}$, but it performs generally well in the acoustic frequency range 0.2 to $0.4 \mathrm{~Hz}$. This range covers the transition from the dominance of 
class III noise events, caused by interacting wind seas and swells, at intermediate frequencies to class I noise generation in which the opposing directions occur within the wind sea thanks to its broad directional distribution toward high frequencies.

This new data clearly shows that ocean currents can also lead to a strong broadening of the wave spectrum induced by wave refraction. Although our measurements are at the bottom in $100 \mathrm{~m}$ depth, the important aspect is really the distance from the sea surface, and open ocean measurements within a few hundred meters of the sea surface would have served just as well because this is where A-G modes dominate. 5

We then considered pressure measurements obtained by hydrophones moored at deep open-ocean sites, and verified that Rayleigh waves dominate acoustic records at large depth. These observations validate key aspects of the theoretical framework used to compute the noise level, and show limitations of the wave model. Our computed noise sources as a function of latitude and longitude and time are freely available, ${ }^{44}$ and now covers the years 1994 to 2012, with an ongoing extension in time. We find that, for wind speeds between 5 and $20 \mathrm{~m} / \mathrm{s}$ and for acoustic frequencies up to four times the wind sea peak frequency (i.e., about $0.6 \mathrm{~Hz}$ in Hawaii and $0.4 \mathrm{~Hz}$ in Kerguelen), the measured noise power is very well correlated with the modeled noise source, proportional to $E^{2}(f) I(f)$. At higher frequencies, the model fails to represent the observed source variability, and two other wave model parameterizations ${ }^{38,39}$ gave even worse results. We attribute these model errors to errors in the estimation of the integral $I(f)$, due to poorly represented processes that may increase the directional wave spreading at high frequencies, possibly associated with wave breaking. ${ }^{17}$

Including the presence of the bottom in our computations, the noise level at both sites, near Hawaii and Kerguelen islands, is found to be higher by 10 to $20 \mathrm{~dB}$ compared to an infinitely deep ocean, for acoustic frequencies below $0.5 \mathrm{~Hz}$. This result contradicts some previous analysis of ocean bottom noise records which ignored that effect, ${ }^{17,24}$ but is consistent with other studies that included it. ${ }^{19}$ Our understanding is that unrealistically broad surface wave spectra had to be assumed to explain noise levels without bottom effects for acoustic frequencies $f_{s}<1 \mathrm{~Hz}$. Typically, ocean waves had to be isotropic for this frequency range, which is not consistent with measurements. ${ }^{10}$

Our computations also show that the properties of the ocean bottom, in particular the presence of pelagic sediments, can strongly modify the noise level. The Kerguelen data, recorded in a vast area of nearly constant depth, reveals the presence of at least four different vertical modes among those expected theoretically in a water layer of $4400 \mathrm{~m}$. A better agreement with the data is obtained when a 500 to $700 \mathrm{~m}$ thick sediment layer is included in the model. Similarly, the shapes of the Hawaii spectra are better explained when modeled with $200 \mathrm{~m}$ of sediments which is consistent with expected sediment thickness in areas where a significant fraction of the noise sources are expected. Sediment thicknesses should thus be included also in seismic noise models.

\section{ACKNOWLEDGMENTS}

The crews of Cotes de la Manche and Marion Dufresne for the FROMVAR and Oha-Sis-Bio cruises made the collection of the data set possible, and the help of Fred Duennebier with the ACO data is gratefully acknowledged. We thank ECMWF for providing analyzed wind fields for this study. F.A. is funded by ERC Grant No. 240009 "IOWAGA" with additional support from the U.S. National Ocean Partnership Program, under Grant No. N00014-10-1-0383, and Labex Mer via grant ANR-10-LABX-19-01. B.H. and R.L. are funded by NSF Grant Nos. OCE-0939570 and OCE-1239637.

\section{APPENDIX A: EXTENDING THE THEORY WITH A SEDIMENT LAYER}

Compared to the homogeneous half-space problem solved by Hasselmann, the addition of a sediment layer on top of the Earth's crust adds four unknowns that are the upgoing and down-going amplitudes of the potential and stream functions. $h_{s}, c_{s p}$, and $c_{s s}$ are, respectively, the sediment layer thickness, compressional wave speed, and shear wave speed. We use the following notations for velocity potentials in water $(\phi)$, in sediments $\left(\phi_{s}\right)$, and in crust $\left(\phi_{c}\right)$ and for stream functions in sediments $\left(\psi_{s}\right)$ and crust $\left(\psi_{c}\right)$,

$$
\begin{aligned}
& \phi=\left(A_{1} e^{i l(z+h)}+A_{2} e^{-i l(z+h)}\right) e^{i \Theta} \\
& \phi_{s}=\left(B_{1} e^{m(z+h)}+B_{2} e^{-m(z+h)}\right) e^{i \Theta} \\
& \psi_{s}=\left(C_{1} e^{n(z+h)}+C_{2} e^{-n(z+h)}\right) e^{i \Theta} \\
& \phi_{c}=D e^{p(z+h)} e^{i \Theta} \\
& \psi_{c}=E e^{q(z+h)} e^{i \Theta} .
\end{aligned}
$$

$l$ is the vertical wave number in water defined in Eq. (3), and $\Theta(\mathbf{K}, s)=\left[\mathbf{K} \cdot \mathbf{x}-2 \pi f_{s} t\right]$ is the phase function. For all these potentials and stream functions, the same wave equation [Eq. (2)] as in the water column applies, with the sound speed in water replaced by the corresponding wave speed. The vertical wavenumbers $m, n, p$, and $q$ are given by equations similar to Eq. (3), e.g.,

$$
m=\sqrt{k^{2}-\frac{\omega_{s}^{2}}{c_{s p}^{2}}} .
$$

The extra layer also adds four more boundary conditions, for the continuity of the normal and tangential velocity and stresses at the sediment-crust interface.

The amplitudes of the velocity potentials and of the stream functions are obtained by solving the eighth order linear system $\mathbf{N X}=\mathbf{B}$ where $\mathbf{X}$ is the state vector, $\mathbf{X}^{T}=\left(A_{1}, A_{2}\right.$, $\left.B_{1}, B_{2}, C_{1}, C_{2}, D, E\right)$ and $\mathrm{B}$ is the forcing term: $B^{T}=\left(\left(i P / p_{w} w_{s}\right), 0,0,0,0,0,0,0\right) . \mathbf{X}^{T}$ and $\mathbf{B}^{T}$ are the transposes of $\mathbf{X}$ and $\mathbf{B}$.

Defining $r_{s}=\omega_{s}^{2}-2 k^{2} c_{s s}^{2}$, and $r_{c}=\omega_{s}^{2}-2 k^{2} c_{c s}^{2}$, the matrix is 


$$
\mathrm{N}=\left(\begin{array}{cccccccc}
e^{i l h} & e^{-i l h} & 0 & 0 & 0 & 0 & 0 & 0 \\
-i l & i l & m & -m & i K & i K & 0 & 0 \\
i w_{s} \rho_{w} & i w_{s} \rho_{w} & -\rho_{s} r_{s} \frac{i}{w_{s}} & -\rho_{s} r_{s} \frac{i}{w_{s}} & -2 \rho_{s} c_{s s}^{2} \frac{n K}{w_{s}} & -2 \rho_{s} c_{s s}^{2} \frac{n K}{w_{s}} & 0 & 0 \\
0 & 0 & -2 K m c_{s s}^{2} & 2 K m c_{s s}^{2} & i r_{s} & i r_{s} & 0 & 0 \\
0 & 0 & i K e^{-m h_{s}} & i K e^{m h_{s}} & -n e^{-n h_{s}} & n e^{n h_{s}} & -i K e^{-p h_{s}} & q e^{-q h_{s}} \\
0 & 0 & -m e^{-m h_{s}} & m e^{m h_{s}} & -i K e^{-n h_{s}} & -i K e^{n h_{s}} & p e^{-p h_{s}} & i K e^{-q h_{s}} \\
0 & 0 & i \rho_{s} r_{s} e^{-m h_{s}} & i \rho_{s} r_{s} e^{m h_{s}} & 2 K n \rho_{s} c_{s s}^{2} e^{-n h_{s}} & -2 K n \rho_{s} c_{s s}^{2} e^{n h_{s}} & -i \rho_{c} r_{c} e^{-p h s} & -2 K q \rho_{c} c_{c s}^{2} e^{-q h_{s}} \\
0 & 0 & 2 K m c_{s s}^{2} e^{-m h_{s}} & -2 K m c_{s s}^{2} e^{m h_{s}} & -i r_{s} e^{-n h_{s}} & -i r_{s} e^{n h_{s}} & -2 K c_{c s}^{2} \rho_{c} e^{-p h_{s}} & i r_{c} \frac{\rho_{c}}{\rho_{s}} e^{-q h_{s}}
\end{array}\right)
$$

We obtain the ground displacement amplitude as a function of the amplitude of the sea surface pressure $\delta=G P$. $G$ is the surface pressure to ground displacement transfer function. In practice this is given by $\partial \phi / \partial z=w=\partial \delta / \partial t$ at $z=-h$

$$
G=\frac{l}{P \omega_{s}}\left(A_{2}-A_{1}\right) .
$$

As $A_{1}$ and $A_{2}$ are proportional to $P, G$ is in fact independent of $P$. For a fixed wave number $K$, there is at least one angular frequency for which the matrix is singular. Assuming that these singularities are simple, we can write $G=\left|G^{\prime}\right| /\left(\omega_{s}^{2}-\omega_{r}^{2}\right)$. For a forcing that varies slowly on the scale of the seismic period $2 \pi / \omega_{s}$, and provided that the forcing spectrum is continuous in spectral space, using Eqs. (8) and (12) provides us with the rate of change of the ground displacement spectrum, ${ }^{8}$

$$
\frac{\partial F_{\delta}\left(K_{x}, K_{y}\right)}{\partial t}=S_{\mathrm{DF}}\left(K_{x}, K_{y}\right)=\frac{\pi\left|G^{\prime}\right|^{2}}{2 \omega_{r}^{2}} F_{p 2, \text { surf }}\left(k_{x}, k_{y}, \omega_{s}\right) .
$$

The seismic spectrum is given by integrating the sources along propagation rays, referenced by the curvilinear abscissa $s$. The source of ground displacement variance per unit of propagation distance can be written as,

$$
\begin{aligned}
S_{\mathrm{DF}}\left(\omega_{s}\right) & =\frac{K(s) S_{\mathrm{DF}}\left(K_{x}, K_{y}\right)}{V^{2}} \\
& =\frac{4 \pi^{2} f_{s} a_{j}^{2}}{c_{c s}^{5} \rho_{s}^{2}} F_{p 2, \operatorname{surf}\left(k_{x}, k_{y}, \omega_{s}\right)}
\end{aligned}
$$

where $V$ is the group speed of the seismic waves and $a_{j}$ is a dimensionless coefficient that depends on $\omega_{s} h / c_{w}$ and the seismic mode index $j$

$$
a_{j}^{2}=\frac{c_{c s}^{2} \rho_{c}^{2} K_{j}\left|G_{j}^{\prime}\right|^{2}}{4 V_{j}^{2} \omega_{s}^{3}} .
$$

Equivalently the noise source can be expressed for the pressure at the ocean bottom, instead of the vertical ground displacement. This is,

$$
\begin{aligned}
S_{\mathrm{DF}, \mathrm{PB}}\left(\omega_{s}\right) & =\frac{K(s) S_{\mathrm{DF}, \mathrm{PB}}\left(K_{x}, K_{y}\right)}{V^{2}} \\
& =\frac{32 \pi^{4} f_{s}^{5} a_{\mathrm{PB}, j}^{2} \rho_{w}^{2}}{c_{c s}^{3} \rho_{c}^{2}} F_{p 2, \operatorname{sur}\left(k_{x}, k_{y}, \omega_{s}\right)},
\end{aligned}
$$

with a dimensionless coefficient $a_{\mathrm{PB}, j}$

$$
a_{\mathrm{PB}, j}^{2}=\frac{c_{c s}^{3} \rho_{c}^{2} K_{j}\left|G_{j}^{\prime}\right|^{2}\left(2 \pi f_{s}\right)^{4} \sin ^{2}(l h)}{l^{2} \cos (l h) \rho_{w}^{2} 4 V_{j}^{2} \omega_{s}^{3}} .
$$

\section{APPENDIX B: GENERAL EXPRESSION FOR EVANESCENT MODES}

In the incompressible limit, and for finite depth and undeformable bottom, the pressure spectrum at the elevation $z$ associated with the evanescent modes is $F_{p 1 D g}\left(f_{2}, z\right)$, and it is given by

$$
\begin{aligned}
& \frac{\cosh ^{2}\left(k_{2} h\right)}{\cosh ^{2}\left[k_{2}(z+k)\right]} F_{p 1 \mathrm{Dg}}\left(f_{2}, z\right) \\
& =\int_{\mathbf{k}_{2}} F_{p 3 D}\left(\mathbf{k}_{2}, f_{2}\right) \mathrm{d} \mathbf{k}_{2} \\
& =\rho_{w}^{2} \int_{\mathbf{k}} \int_{\mathbf{k}^{\prime}} D_{z}^{2} F(\mathbf{k}) F\left(\mathbf{k}^{\prime}\right) \delta\left(f+f^{\prime}-f_{2}\right) \mathrm{d} \mathbf{k} \mathrm{d} \mathbf{k}^{\prime} \\
& =\rho_{w}^{2} \int_{-f_{2}}^{f_{2}} \int_{0}^{2 \pi} \int_{0}^{2 \pi} D_{z}^{2} F(f, \theta) F\left(f^{\prime}, \theta^{\prime}\right) \mathrm{d} \theta \mathrm{d} \theta^{\prime} \mathrm{d} \Delta_{f},
\end{aligned}
$$

with a coupling coefficient $D_{z}$ for the elevation amplitudes given by ${ }^{23}$

$$
\begin{aligned}
D_{z}\left(\mathbf{k}, \mathbf{k}^{\prime}\right)= & \frac{g^{2}}{\sigma \sigma^{\prime}}\left\{\left[\frac{\sigma^{2} \sigma^{\prime 2}}{g^{2}}-\mathbf{k} \cdot \mathbf{k}^{\prime}\right]\right. \\
& \left.-\frac{0.5}{\left(\mathrm{~s} \sigma+s^{\prime} \sigma^{\prime}\right)}\left(\frac{\sigma k^{\prime 2}}{\cosh ^{2}\left(k^{\prime} h\right)}+\frac{\sigma^{\prime} k^{2}}{\cosh ^{2}(k h)}\right)\right\} .
\end{aligned}
$$

${ }^{1}$ T. H. C. Herbers and R. T. Guza, "Wind-wave nonlinearity observed at the sea floor. Part I: forced-wave energy," J. Phys. Oceanogr. 21, 1740-1761 (1991).

${ }^{2}$ C. S. Cox and D. C. Jacobs, "Cartesian diver observations of double frequency pressure fluctuations in the upper levels of the ocean," Geophys. Res. Lett. 16, 807-810, doi:10.1029/GL016i008p00807 (1989). 
${ }^{3}$ S. C. Webb, "Broadband seismology and noise under the ocean," Rev. Geophys. 36, 105-142, doi:10.1029/97RG02287 (1998).

${ }^{4}$ M. Obrebski, F. Ardhuin, E. Stutzmann, and M. Schimmel, "How moderate sea states can generate loud seismic noise in the deep ocean," Geophys. Res. Lett. 39, L11601 (2012).

${ }^{5}$ F. Ardhuin and T. H. C. Herbers, "Double-frequency noise generation by surface gravity waves in finite depth: Gravity, acoustic and seismic modes," J. Fluid Mech. 716, 316-348 (2013).

${ }^{6}$ R. Stoneley, "The effect of the ocean on Rayleigh waves," Mon. Not. R. Astron. Soc. Geophys. Suppl. 1, 349-356 (1926).

7M. S. Longuet-Higgins, "A theory of the origin of microseisms," Philos. Trans. R. Soc. London, Ser. A 243, 1-35 (1950).

${ }^{8} \mathrm{~K}$. Hasselmann, "A statistical analysis of the generation of microseisms," Rev. Geophys. 1, 177-210, doi:10.1029/RG001i002p00177 (1963).

${ }^{9}$ S. Kedar, M. Longuet-Higgins, F. W. N. Graham, R. Clayton, and C. Jones, "The origin of deep ocean microseisms in the north Atlantic ocean," Proc. R. Soc. London, Ser. A 464, 1-35 (2008).

${ }^{10}$ F. Ardhuin, E. Stutzmann, M. Schimmel, and A. Mangeney, "Ocean wave sources of seismic noise,” J. Geophys. Res. 116, C09004, doi:10.1029/ 2011JC006952 (2011).

${ }^{11}$ E. Stutzmann, M. Schimmel, and F. Ardhuin, "Modeling long-term seismic noise in various environments," Geophys. J. Int. 191, 707-722 (2012).

${ }^{12}$ G. Nolet and L. M. Dorman, "Waveform analysis of Scholte modes in ocean sediment layers," Geophys. J. Int. 125, 385-396 (1996).

${ }^{13} \mathrm{~B}$. Hughes, "Estimates of underwater sound (and infrasound) produced by nonlinearly interacting ocean waves," J. Acoust. Soc. Am. 60, 1032-1039 (1976).

${ }^{14}$ S. P. Lloyd, "Underwater sound from surface waves according to the Lighthill-Ribner theory," J. Acoust. Soc. Am. 69, 425-435 (1981).

${ }^{15}$ A. C. Kibblewhite and C. Y. Wu, "The generation of infrasonic ambient noise in the ocean by nonlinear interactions of ocean surface waves," J. Acoust. Soc. Am. 85, 1935-1945 (1989).

${ }^{16}$ A. C. Kibblewhite and K. C. Ewans, Wave Interactions as a SeismoAcoustic Source (Springer, Berlin, Germany, 1996).

${ }^{17}$ F. K. Duennebier, R. Lukas, E.-M. Nosal, J. Aucan, and R. A. Weller, "Wind, waves, and acoustic background levels at station ALOHA," J. Geophys. Res. 117, C03017, doi:10.1029/2011JC007267 (2012).

${ }^{18}$ S. C. Webb and C. S. Cox, "Observation and modeling of seafloor microseisms," J. Geophys. Res. 91, 7343-7358, doi:10.1029/JB091iB07p07343 (1986).

${ }^{19}$ S. C. Webb, "The equilibrium oceanic microseism spectrum," J. Acoust. Soc. Am. 92, 2141-2158 (1992).

${ }^{20}$ D. K. Wilson, G. V. Frisk, T. E. Lindstrom, and C. J. Sellers, "Measurement and prediction of ultralow frequency ocean ambient noise off the eastern U.S. coast," J. Acoust. Soc. Am. 113, 3117-3133 (2003).

${ }^{21}$ H. L. Tolman, "A mosaic approach to wind wave modeling," Ocean Modeling 25, 35-47 (2008).

${ }^{22}$ F. Ardhuin, E. Rogers, A. Babanin, J.-F. Filipot, R. Magne, A. Roland, A. van der Westhuysen, P. Queffeulou, J.-M. Lefevre, L. Aouf, and F. Collard, "Semi-empirical dissipation source functions for wind-wave models: Part I. Definition, calibration, and validation" J. Phys. Oceanogr. 40, 1917-1941 (2010).

${ }^{23} \mathrm{~K}$. Hasselmann, "On the non-linear energy transfer in a gravity wave spectrum, Part 1: General theory," J. Fluid Mech. 12, 481-501 (1962).

${ }^{24}$ W. E. Farrell and W. Munk, "Booms and busts in the deep," J. Phys. Oceanogr. 40, 2159-2169 (2010).
${ }^{25}$ F. Ardhuin, A. Balanche, E. Stutzmann, and M. Obrebski, "From seismic noise to ocean wave parameters: General methods and validation," J. Geophys. Res. 117, C05002, doi:10.1029/2011JC007449 (2012).

${ }^{26}$ K. Hasselmann, T. P. Barnett, E. Bouws, H. Carlson, D. E. Cartwright, K. Enke, J. A. Ewing, H. Gienapp, D. E. Hasselmann, P. Kruseman, A. Meerburg, P. Müller, D. J. Olbers, K. Richter, W. Sell, and H. Walden, "Measurements of wind-wave growth and swell decay during the Joint North Sea Wave Project," Dtsch. Hydrogr. Z. 8(A), 1-95 (1973).

${ }^{27} \mathrm{~F}$. Ardhuin, J. Tournadre, P. Queffelou, and F. Girard-Ardhuin, "Observation and parameterization of small icebergs: Drifting breakwaters in the southern ocean," Ocean Modeling 39, 405-410 (2011).

${ }^{28}$ F. Ardhuin, L. Marié, N. Rascle, P. Forget, and A. Roland, "Observation and estimation of Lagrangian, Stokes, and Eulerian currents induced by wind and waves at the sea surface," J. Phys. Oceanogr. 39, 2820-2838 (2009).

${ }^{29}$ F. Ardhuin, F. Dumas, A.-C. Bennis, A. Roland, A. Sentchev, P. Forget, J. Wolf, F. Girard, P. Osuna, and M. Benoit, "Numerical wave modeling in conditions with strong currents: Dissipation, refraction, and relative wind," J. Phys. Oceanogr. 42, 2101-2120 (2012).

${ }^{30}$ F. Ardhuin and A. Roland, "Coastal wave reflection, directional spreading, and seismoacoustic noise sources," J. Geophys. Res. 117, C00J20, doi:10.1029/2011JC007832 (2012)

${ }^{31}$ T. H. C. Herbers and R. T. Guza, "Nonlinear wave interactions and highfrequency seafloor pressure," J. Geophys. Res. 99, 10035-10048, doi:10.1029/94JC00054 (1994)

${ }^{32}$ H. E. Krogstad and K. Trulsen, "Interpretations and observations of ocean wave spectra," Ocean Dyn. 62, 973-991 (2010).

${ }^{33}$ B. J. Mitchell, "Anelastic structure and evolution of the continental crust and upper mantle from seismic surface wave attenuation," Rev. Geophys. 33, 441-462, doi:10.1029/95RG02074 (1995).

${ }^{34} \mathrm{~F}$. Abramovici, "Diagnostic diagrams and transfer functions for oceanic wave-guides," Bull. Seismol. Soc. Am. 58, 426-456 (1968).

${ }^{35} \mathrm{H}$. Bradner, L. G. de Jerphanion, and R. Langlois, "Ocean microseism measurements with a neutral buoyancy free floating midwater seismometer," Bull. Seismol. Soc. Am. 60, 1139-1150 (1970).

${ }^{36}$ J. H. G. M. Alves, M. L. Banner, and I. R. Young, "Revisiting the PiersonMoskowitz asymptotic limits for fully developed wind waves," J. Phys. Oceanogr. 33, 1301-1323 (2003).

${ }^{37} \mathrm{~A}$. Benetazzo, "Measurements of short water waves using stereo matched image sequences," Coastal Eng. 53, 1013-1032 (2006).

${ }^{38}$ J. Bidlot, P. Janssen, and S. Abdalla, "A revised formulation for ocean wave dissipation in CY25R1," Technical Report Memorandum R60.9/JB/ 0516, Research Department, ECMWF, Reading, United Kingdom (2005).

${ }^{39}$ H. L. Tolman and D. Chalikov, "Source terms in a third-generation wind wave model," J. Phys. Oceanogr. 26, 2497-2518 (1996).

${ }^{40} \mathrm{O}$. M. Phillips, "Spectral and statistical properties of the equilibrium range in wind-generated gravity waves," J. Fluid Mech. 156, 505-531 (1985).

${ }^{41} \mathrm{M}$. V. Trevorrow and T. Yamamoto, "Summary of marine sedimentary shear modulus and acoustic speed profile results using a gravity wave inversion technique," J. Acoust. Soc. Am. 90, 441-456 (1991).

${ }^{42}$ D. Divins, "Total sediment thickness of the world's oceans and marginal seas," Technical Report, NOAA National Geophysical Data Center, Boulder, CO (2003).

${ }^{43}$ E. L. Hamilton, "Shear-wave velocity versus depth in marine sediments: A review," Geophysics 41, 985-996 (1976).

${ }^{44}$ IOWAGA database, available at http://tinyurl.com/iowagaftp/iowaga/ SISMO (Last viewed November 14, 2012). 